\title{
Insertional mutagenesis in a HER2-positive breast cancer model reveals ERAS as a driver of cancer and therapy resistance
}

\author{
Gerjon J. Ikink $\mathbb{D}^{1} \cdot$ Mandy Boer $^{1} \cdot$ Elvira R. M. Bakker ${ }^{1}$ - Annabel Vendel-Zwaagstra ${ }^{1} \cdot$ Chris Klijn $^{2}$ - Jelle ten Hoeve ${ }^{3}$. \\ Jos Jonkers ${ }^{2} \cdot$ Lodewyk F. Wessels $^{3} \cdot$ John Hilkens $^{1}$
}

Received: 6 April 2017 / Revised: 29 October 2017 / Accepted: 31 October 2017 / Published online: 12 January 2018

(c) The Author(s) 2018. This article is published with open access

\begin{abstract}
Personalized medicine for cancer patients requires a deep understanding of the underlying genetics that drive cancer and the subsequent identification of predictive biomarkers. To discover new genes and pathways contributing to oncogenesis and therapy resistance in HER2+ breast cancer, we performed Mouse Mammary Tumor Virus (MMTV)-induced insertional mutagenesis screens in ErbB2/cNeu-transgenic mouse models. The screens revealed 34 common integration sites (CIS) in mammary tumors of MMTV-infected mice, highlighting loci with multiple independent MMTV integrations in which potential oncogenes are activated, most of which had never been reported as MMTV CIS. The CIS most strongly associated with the ErbB2-transgenic genotype was the locus containing Eras (ES cell-expressed Ras), a constitutively active RASfamily GTPase. We show that upon expression, Eras acts as a potent oncogenic driver through hyperactivation of the PI3K/ AKT pathway, in contrast to other RAS proteins that signal primarily via the MAPK/ERK pathway and require upstream activation or activating mutations to induce signaling. We additionally show that ERAS synergistically enhances HER2induced tumorigenesis and, in this role, can functionally replace ERBB3/HER3 by acting as a more powerful activator of PI3K/AKT signaling. Although previously reported as pseudogene in humans, we observed ERAS RNA and protein expression in a substantial subset of human primary breast carcinomas. Importantly, we show that ERAS induces primary resistance to the widely used HER2-targeting drugs Trastuzumab (Herceptin) and Lapatinib (Tykerb/Tyverb) in vivo, and is involved in acquired resistance via selective upregulation during treatment in vitro, indicating that ERAS may serve as a novel clinical biomarker for PI3K/AKT pathway hyperactivation and HER2-targeted therapy resistance.
\end{abstract}

\section{Introduction}

HER $2+$ breast cancer is highly aggressive and afflicted patients have a poor clinical outcome [1]. Treatment with the humanized monoclonal antibody against ERBB2, Trastuzumab, or the small molecule kinase inhibitor

Electronic supplementary material The online version of this article (https://doi.org/10.1038/s41388-017-0031-0) contains supplementary material, which is available to authorized users.

John Hilkens

j.hilkens@nki.nl

1 Division of Molecular Genetics, The Netherlands Cancer Institute, Amsterdam, The Netherlands

2 Division of Molecular Pathology, The Netherlands Cancer Institute, Amsterdam, The Netherlands

3 Division of Molecular Carcinogenesis, The Netherlands Cancer Institute, Amsterdam, The Netherlands
Lapatinib, typically combined with chemotherapy, significantly improves the clinical outcome [2,3], but inherent and acquired resistance to both drugs are prevalent [4]. To study HER2 + breast cancer, various transgenic mouse strains have been raised as models for the human disease [5-8], in which mammary tumors stochastically arise that closely recapitulate the histopathological and molecular features of HER2 + human breast carcinomas [9, 10].

Insertional mutagenesis in mouse models is an effective method to discover novel genes involved in breast cancer development. We and others have previously identified a series of novel candidate cancer genes using MMTVmediated insertional mutagenesis in mice [11-15]. MMTV causes a high incidence of murine mammary carcinomas by random insertion of its proviral DNA into the host DNA, which can activate adjacent proto-oncogenes [16-18]. The genomic localization of the proviral insertion can easily be determined using the technologies developed in our 
laboratory $[11,14]$, thus allowing the identification of candidate oncogenes.

We performed high-throughput insertional mutagenesis screens in MMTV-infected ErbB2-transgenic mice to identify genes and pathways that collaborate with ERBB2overexpression in mammary tumorigenesis. Combined results of a screen using the classical splinkerette PCR method [11] and a screen using our Shear-Splink technology followed by deep sequencing [14, 19], revealed 34 common integration sites (CIS) associated with putative oncogenes, of which the majority has not previously been reported as an MMTV CIS.

We found the Eras gene as the most common MMTVproviral target specific to ErbB2-induced mammary tumors.

Table 1 CISs found in the insertional mutagenesis screens in tumors from MMTV-cNeu mice

\begin{tabular}{|c|c|c|c|c|c|c|}
\hline Locus & Chr. & $\begin{array}{l}\text { Tumors with } \\
\text { insertion } \\
\text { (classical } \\
\text { setup) }\end{array}$ & $\begin{array}{l}\text { Tumors with } \\
\text { insertion } \\
\text { (Shear-Splink) }\end{array}$ & $\begin{array}{l}\text { Known } \\
\text { MMTV } \\
\text { target }\end{array}$ & $\begin{array}{l}\text { CIS in } \\
\text { RTCGD } \\
\text { (retrovirus) }\end{array}$ & $\begin{array}{l}\text { CIS in RTCGD } \\
\text { (transposon) }\end{array}$ \\
\hline Wnt1/Wnt10b & $15 \mathrm{~F} 1$ & 16 & 14 & Yes & 28 & - \\
\hline Eras & XA1.1 & 8 & 11 & Yes & 6 & 2 \\
\hline Fgf3/Fgf4 & $7 \mathrm{~F} 5$ & 7 & 9 & Yes & 38 & - \\
\hline Fgf8 & $19 \mathrm{C} 3$ & 5 & 4 & Yes & 15 & - \\
\hline Wnt3a & $11 \mathrm{~B} 1.3$ & 5 & 4 & Yes & 10 & - \\
\hline Irs4 & $\mathrm{XF} 2$ & 3 & 4 & Yes & - & - \\
\hline $\begin{array}{l}\text { Gm26870 } \\
\text { (lincRNA)/ } \\
\text { Mir101c }\end{array}$ & 9A1 & 1 & 6 & No & - & - \\
\hline Metrnl/Ptchd3 & $11 \mathrm{E} 2$ & 3 & 3 & Yes & - & - \\
\hline $\begin{array}{l}\text { Gm10800/ } \\
\text { Gm10801 }\end{array}$ & $2 \mathrm{E} 1$ & 1 & 5 & No & - & - \\
\hline Lrfn5 & $12 \mathrm{C} 1$ & 1 & 4 & No & - & - \\
\hline Astn2 & $4 \mathrm{C} 1$ & 2 & 2 & Yes & 2 & - \\
\hline Fgfr2 & $7 \mathrm{~F} 3$ & 2 & 2 & Yes & - & - \\
\hline Map3k8 & $18 \mathrm{~A} 1$ & 1 & 3 & Yes & 16 & - \\
\hline Wnt3 & $11 \mathrm{E} 1$ & 1 & 3 & Yes & 14 & - \\
\hline Gpc6/Gpc5 & $14 \mathrm{E} 4$ & 3 & 1 & No & - & - \\
\hline Zfp521 & 18A1 & 3 & 1 & No & 7 & - \\
\hline Sfil & $11 \mathrm{~A} 1$ & 1 & 2 & Yes & - & - \\
\hline Fgfr1 & $8 \mathrm{~A} 2$ & 2 & 1 & Yes & - & - \\
\hline Nova1 & 12B3 & 2 & 1 & No & - & - \\
\hline Ywhaq & 12A 1.3 & 2 & 1 & No & - & - \\
\hline Pdgfrb & $18 \mathrm{E} 1$ & 0 & 2 & Yes & - & - \\
\hline Arf6 & $12 \mathrm{C} 2$ & 2 & 0 & No & 4 & - \\
\hline $\begin{array}{l}\text { Arpm1 } \\
\text { (Actrt3) }\end{array}$ & $3 \mathrm{~A} 3$ & 2 & 0 & No & - & - \\
\hline Btg1 & $10 \mathrm{C} 3$ & 2 & 0 & No & - & - \\
\hline Ctif & $18 \mathrm{E} 3$ & 2 & 0 & No & - & - \\
\hline Dnah1 & $14 \mathrm{~B}$ & 2 & 0 & No & - & - \\
\hline Fhit & $14 \mathrm{~A} 1$ & 2 & 0 & No & - & 2 \\
\hline Id 2 & 12A1.3 & 2 & 0 & No & 3 & - \\
\hline Mid1 & XF5 & 2 & 0 & No & 7 & - \\
\hline Mme & $3 \mathrm{E} 1$ & 2 & 0 & No & - & - \\
\hline Osbpl5 & $7 \mathrm{~F} 5$ & 2 & 0 & No & - & - \\
\hline Osbpl8 & 10D1 & 2 & 0 & No & - & - \\
\hline Runx1 & $16 \mathrm{C} 4$ & 2 & 0 & No & 9 & - \\
\hline Slc25a25 & $2 \mathrm{~B}$ & 2 & 0 & No & - & - \\
\hline
\end{tabular}

Chr. chromosome, - no CIS reported 

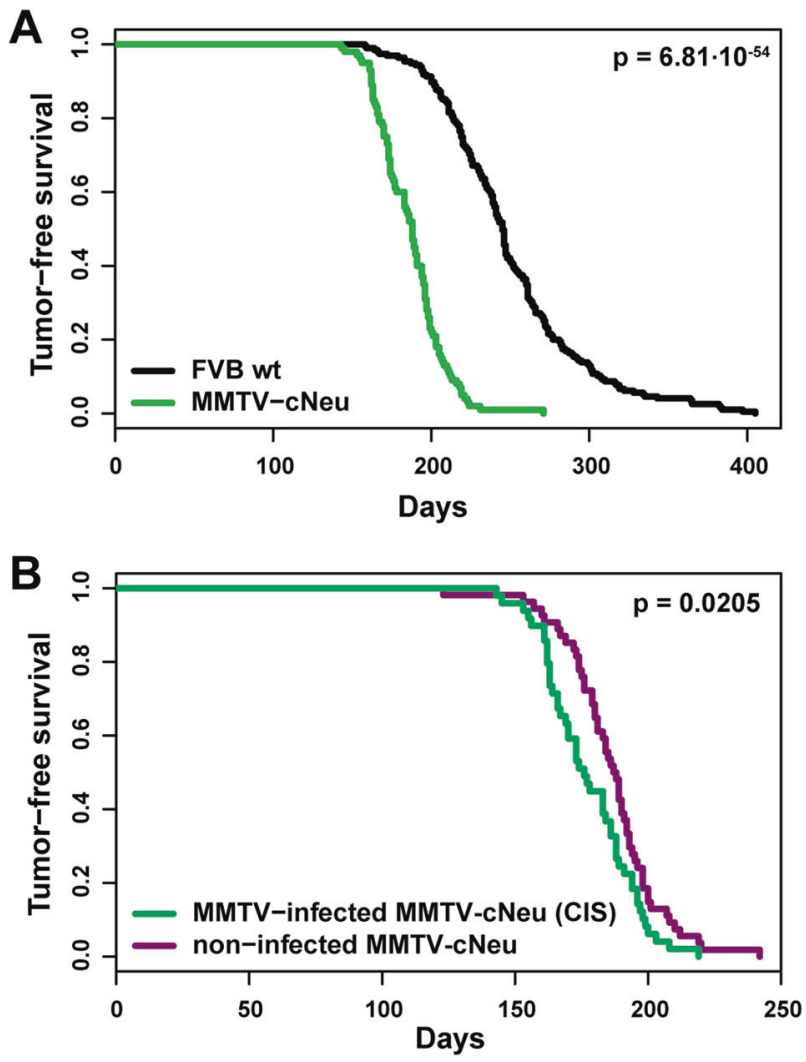

Fig. 1 Impact of MMTV infection on tumor free survival of $M M T V$ $c N e u$ and wildtype FVB mice. a, b Kaplan-Meier curves of the MMTV-infected MMTV-cNeu mice $(n=100)$ compared to the MMTV-infected parental FVB wildtype strain $(n=195)$ (a) and MMTV-infected MMTV-cNeu mice with tumors carrying insertions in common insertion sites (CIS) $(n=49)$ compared to non-infected $M M T V$ - $c N e u$ mice $(n=54)$ (b). Statistical significance of differences in latency was determined by the pairwise log-rank test applying the survdiff function in $\mathrm{R}$ ( $p$-values in upper-right corner). All mice eventually developed tumors and only mice that prematurely died from other causes were excluded

ERAS is a member of the RAS-family of small GTPases containing amino acid sequences identical to those present in constitute active mutants of HRAS (e.g., HRasV12) and is therefore in a permanently active (GTP-bound) state [20, 21]. Mouse Eras is only expressed in embryonic stem (ES) cells and appears to be responsible for the tumor-like growth properties of ES cells when growing ectopically [21]. In addition, ERAS was reported to be required for somatic cell reprogramming to generate induced pluripotent stem (iPS) cells and the differentiation of ES cells into specific lineage cells [22-24]. In all these processes, the activation of the PI3K/AKT pathway by ERAS has been implicated, in contrast to the MAPK/ERK pathway generally activated by other RAS-family members.

Here, we report that ERAS is an oncogenic driver that acts synergistically with $E r b B 2$ in mammary tumorigenesis. Moreover, we show that ERAS expression occurs in a sizeable fraction of human HER2 + breast cancers. Finally, ERAS confers resistance to the HER2-targeted therapeutic agents Trastuzumab and Lapatinib through potent PI3K/ AKT pathway activation.

\section{Results}

\section{MMTV insertional mutagenesis in ErbB2+ predisposed background}

We performed high-throughput sequencing of MMTV integration sites in mammary tumors obtained from MMTV-infected FVB mice, transgenic for rat ErbB2 (neu) driven by the MMTV-promoter (MMTV-cNeu strain). We employed both the classical splinkerette PCR method and the more advanced "Shear-Splink" technology combined with the Gaussian Kernel Convolution framework in the Insertional Mutagenesis Database (iMDB; http://imdb.nki. nl) pipeline. To discriminate MMTV insertions that activate genes driving tumorigenesis from passenger insertions, we identified the CISs among independent tumors. In total, the screens yielded 34 CISs, of which $23(68 \%)$ were found in both screens (Table 1). Twenty CISs have not been previously identified as an MMTV target, of which 14 have additionally not even been reported as CIS in the Retrovirus and Transposon tagged Cancer Gene Database (RTCGD; http://variation.osu.edu/rtcgd/) [25] (Table 1).

We compared the results in the MMTV-cNeu transgenic mice obtained with the "Shear-Splink" setup in the present screen with the results from an identical screen in the parental FVB wildtype strain published earlier by our group [14]. Although the median tumor latency of the MMTVinfected MMTV-cNeu transgenic mice (188 days) was strongly decreased when compared to wildtype FVB mice infected with MMTV (245 days) (Fig. 1a), there was no significant difference in tumor latency between MMTVinfected and uninfected $M M T V-c N e u$ mice. However, only $39 \%$ (59/151) of the independent tumors from MMTVinfected MMTV-cNeu mice contained an MMTV insertion belonging to a CIS in MMTV-cNeu mice, compared to $93 \%$ in MMTV-infected FVB wildtype mice [14]. This indicates that tumorigenesis driven by the predisposed background is in strong competition with tumorigenesis driven by MMTV insertional mutagenesis. When comparing only the MMTVinfected MMTV-cNeu mice with tumors driven by a CIS, the median latency was significantly decreased (176 days) compared to uninfected MMTV-cNeu mice (188 days) (Fig. 1b), suggesting that at least part of the CIS-associated MMTV integrations may accelerate mammary tumorigenesis in MMTV-cNeu mice in collaboration with the ErbB2transgene. 


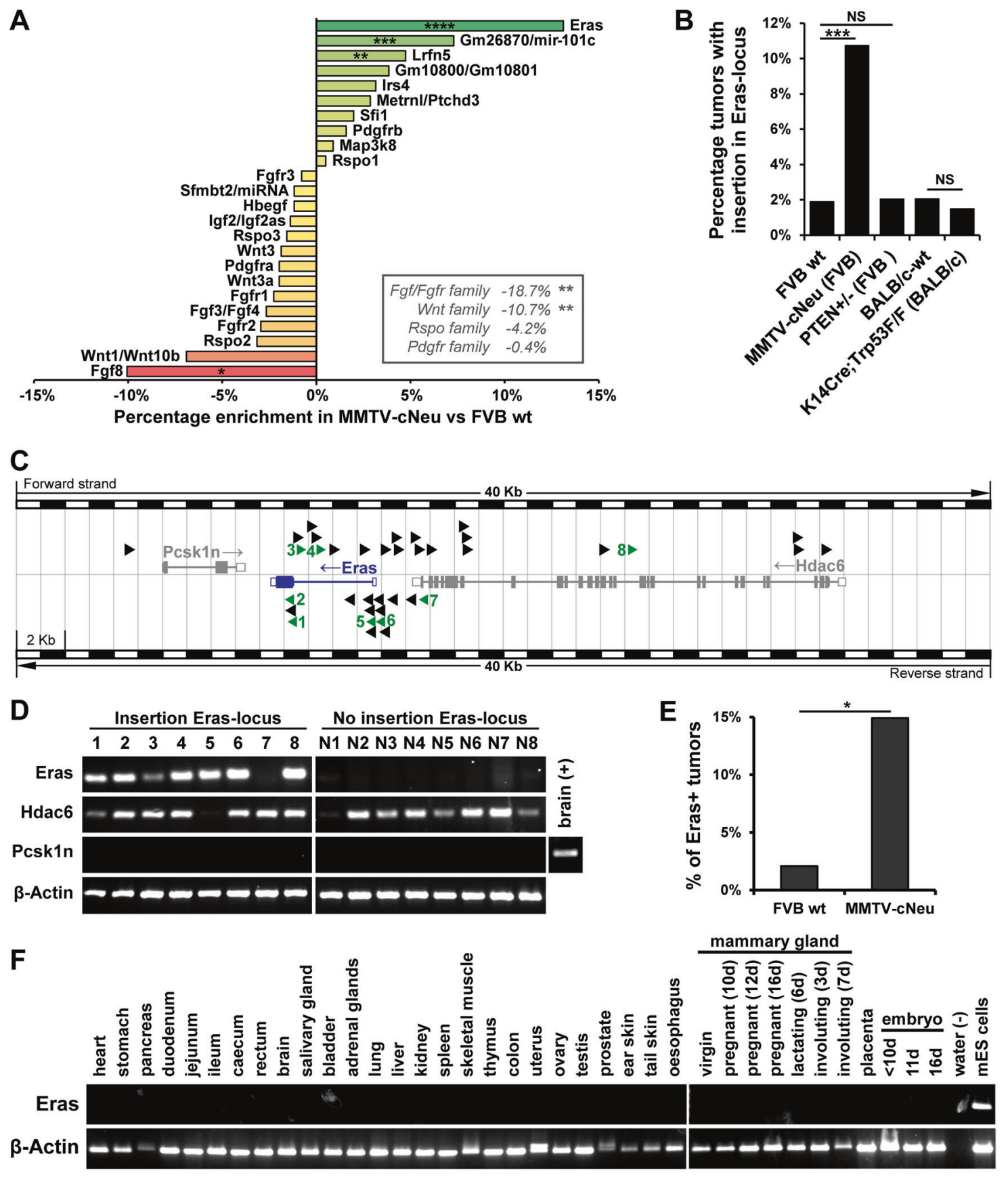

\section{MMTV target Eras is associated with the ErbB2/Neu genotype}

Loci harboring genes of the Wnt, Fgf, and $F g f$-family were among the most frequent CISs (Table 1), but these loci are also frequent hits in wildtype FVB, BALB/c, and other mouse strains $[11,13,14]$, and thus unlikely to be specific to the ErbB2-transgenic (MMTV-cNeu) genotype. Comparing the CIS-frequency in the ErbB2-transgenic FVB mouse models with the CIS-frequency in its parental FVB wildtype strain, revealed even a significantly negative correlation of the Wnt and $F g f / F g f$-family CISs with the MMTV-cNeu genotype $\left(p=7.64 \times 10^{-3}\right.$ and $p=1.48 \times 10^{-3}$, respectively, Fisher's exact test) (Fig. 2a). Three CISs, however, significantly associated with the ErbB2-genotype, including the loci containing Lrfn5 (leucine-rich repeat and fibronectin type III domain-containing 5), the long intergenic non-coding RNA Gm26870 or microRNA-101c (Mir101c), and especially Eras (ES cell-expressed Ras) $(p=3.57 \times$ $10^{-3}, p=1.06 \times 10^{-4}$ and $p=3.08 \times 10^{-7}$, respectively, Fisher's exact test). More CISs may be associated with ErbB2-overexpression, considering that, e.g., the Irs4-locus 
Fig. 2 Eras is an MMTV target associated with the ErbB2/Neu genotype. a Enrichment of MMTV CISs in $c N e u$-transgenic FVB mice compared to FVB wildtype mice, calculated as percentage of all independent tumors in each group. The same analysis for gene families are shown in the lower-right corner. Significance of positive or negative enrichment was determined by a Fisher's exact test and $p$ values are shown as $* p<0.05 ; * * p<0.01 ; * * * p<0.001 ; * * * * p<$ $1 \times 10^{-4}$. See also Supplementary Table 2 . b Percentage of independent tumors with an MMTV insertion in the Eras locus for the mouse strains: FVB wildtype $(n=265), M M T V-c N e u(n=177)$, PTEN + / $(n=196), \mathrm{BALB} / \mathrm{c}$ wildtype $(n=292)$, and K14Cre;Trp53 ${ }^{F / F}(n=$ 202). Statistical significance of differences in relevant comparisons was determined by a Fisher's exact test: NS not significant; ***p $<$ 0.001. c Map of MMTV integrations in the Pcsk1n/Eras/Hdac6-locus on the X-chromosome, showing the gene names (arrow heads indicate orientation), their translated exons (solid rectangles), UTRs (open rectangles), and the interspacing introns (lines). MMTV-proviral insertions are indicated with triangles pointing in the orientation of integration. Green arrow heads represent tumors analyzed in d. d RTPCR expression analysis of Eras, Pcsk1n, and Hdac6 in tumors with integration in the Pcsk1n/Eras/Hdac6-locus, following the numbering of $\mathbf{c}$ and random tumors without integrations in this locus (the rest of the analysis is provided in Supplementary Fig. 1A). PCR-products for $\beta$-actin were used as controls for RNA integrity. e Percentage of ErasmRNA expressing independent MMTV-induced tumors from FVB wildtype mice $(n=48)$ and $c N e u$-transgenic FVB mice $(n=161)$, showing that Eras is significantly more commonly expressed in tumors from $c N e u$-transgenic mice; $p$-value $=0.01973(*)$, determined by a Fisher's exact test. $\mathbf{f}$ Eras-mRNA expression in tissues from BALB/c wildtype mice, including tissues from various stages of mammary gland development and embryogenesis (showing days), determined by RT-PCR. A sample of murine ES cells was used as positive control and $\beta$-actin expression was assessed as control for RNA integrity

here only shows a trend towards enrichment in the ErbB2 genotype (Fig. 2a), whereas we recently reported that IRS4 collaborates with ERBB2 in tumorigenesis [15, 26]. When relating the MMTV integration data reported here with those of our previously published screens [11, 14], integrations in the Eras locus were far more common in the $M M T V-c N e u$ genotype compared to the FVB wildtype strain $\left(p=1.28 \times 10^{-4}\right.$, Fisher's exact test), and also compared to the BALB/c wildtype, K14Cre;Trp5 $3^{F / F}$ (BALB/c background), and $P T E N^{+-}$mice (FVB background) (Fig. $2 b)$. This indicates that integrations in the Eras locus provides a selective growth advantage especially in ErbB2 transgenic mice.

On the basis of the genomic location and orientation of the MMTV-proviral integrations in the Eras locus, Eras is the most likely gene candidate to be transcriptionally activated (Fig. 2c), but the locus also contains the Pcskln (proprotein convertase subtilisin/kexin type 1 inhibitor) and Hdac6 (histone deacetylase 6) genes that could also be transcriptionally activated by the MMTV insertions. To determine the actual MMTV target gene(s), we performed a side-by-side analysis of Pcsk1n, Eras, and Hdac6 mRNA expression in eight $M M T V$-cNeu tumors with and 32 tumors without MMTV-proviral insertion in the Eras locus using
RT-PCR (Fig. 2d; Supplementary Fig. 1A). Pcsk1n was not expressed in any of the tumors, excluding this gene as the MMTV target. Hdac6 mRNA was present in all but one of the tumors from both groups, regardless of the presence of an MMTV insertion in the Eras locus. Moreover, also MMTV-induced mammary tumors from FVB wildtype mice and spontaneous tumors from ErbB2 transgenic mice were almost all positive for Hdac6 mRNA (Supplementary Fig. $1 \mathrm{~B}, \mathrm{C}$ ), indicating that expression of Hdac6 is rather ubiquitous in mouse mammary tumors and not correlated to MMTV integrations in the Eras locus. Only Eras expression was clearly correlated with MMTV integration in the locus $\left(p=1.023 \times 10^{-4}\right.$, Fisher's exact test), showing expression in all but one tumor with an MMTV integration in the Eras locus, whereas no clear expression was observed in tumors without integration.

As our stringent method of assigning integration sitesdesigned to limit false-positive findings-inherently produces false-negatives in our screens, the number of integrations we report is likely an underestimation. Therefore, we compared Eras-gene expression in a panel of 161 tumors from MMTV-infected ErbB2 transgenic FVB mice (including those in Fig. 2d and Supplementary Fig. 1A) with the expression in a panel of 48 tumors from MMTVinfected wildtype FVB mice. In accordance with our previous results, we found that Eras was significantly more frequently expressed in MMTV-induced tumors from ErbB2-transgenic mice (15\%) compared to wildtype mice (2\%) (Fig. 2e). This strongly suggests that Eras specifically collaborates with $E r b B 2$ in mammary tumorigenesis.

Importantly, we observed no Eras expression during any stage of murine mammary gland development, nor in any other mouse tissue or during embryogenesis (from $\sim \mathrm{E} 9$ onwards). In line with previous reports [21], we exclusively observed expression of the gene in ES cells, indicating that Eras is strictly regulated at the transcriptional level (Fig. 2f).

\section{ERAS stimulates cell proliferation synergistically with ERBB2}

To investigate the biological basis of the revealed oncogenic collaboration between Eras and ErbB2, we ectopically expressed ERAS in the human HER2+ (amplified wildtype ErbB2) breast cancer cell line SKBR3. We observed a strongly increased cell proliferation rate in cells transduced with ERAS compared to vector controls (Fig. 3a; Supplementary Fig. 2A). To assess the contribution of Eras and ErbB2, separately and in combination, we compared the proliferation rates of the spontaneously immortalized normal mammary epithelial cell line NMuMG transduced with Eras and ErbB2 individually, Eras and ErbB2 combined, or the empty vector control under various growth conditions 
Fig. 3 ERAS promotes cell proliferation synergistically with ERBB2. a Growth curves based on Crystal Violet staining of SKBR3 cells ectopically expressing ERAS compared to vector control cells, showing mean \pm SD cell density, relative to day $0(n=3) \quad(25,000$ cells per well, 12-wells plates). Micrographs of these cells at day 9 are provided in Supplementary Fig. 2A. b Western blot showing human and murine ERAS protein in NMuMG cells stably transduced with human or murine ERAS (hERAS or mEras, respectively), $E r b B 2$ alone, $E r b B 2$ and ERAS, or the empty vector. $\beta$-actin was used as loading control. This anti-ERAS antibody did not react with HRas or KRas (see Supplementary Fig. 2B). These results were confirmed with another antibody for ERAS (see Supplementary Fig. 2C). c Growth curves of NMuMG cells as in $\mathbf{b}$ under normal growth conditions $(10 \%$ FBS + insulin), without insulin or under low serum conditions (0.5\% FBS), based on Crystal Violet staining $(30,000$ cells per well, 12-wells plate). Plotted values are mean $\pm \mathrm{SD}$, relative to day $0(n=3)$
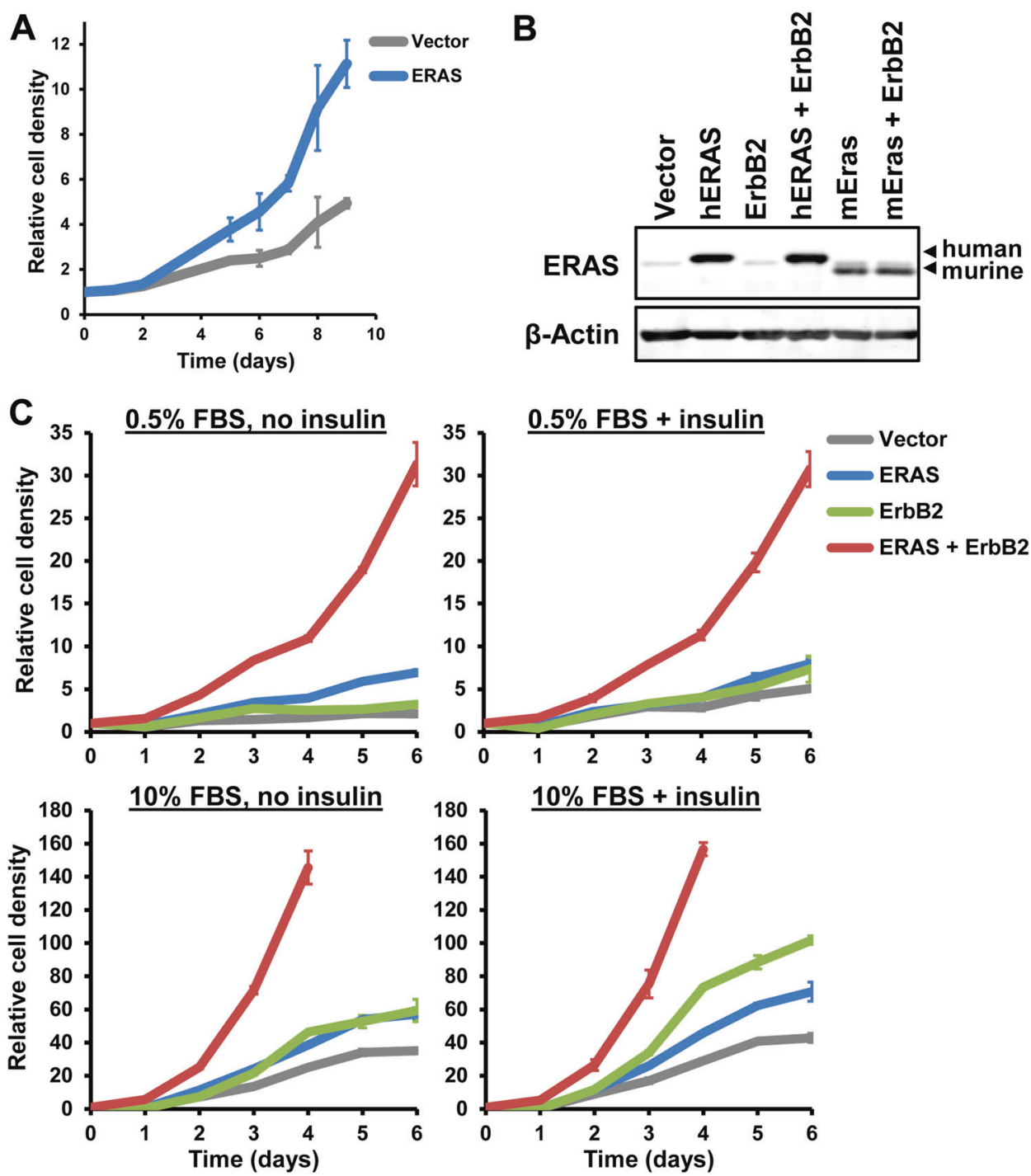

(Fig. 3b, c; Supplementary Fig. 2B, C). Under all growth conditions, Eras-expressing NMuMG cells showed a higher cell proliferation rate than the vector control. Moreover, cells expressing Eras appeared to be independent of insulin for growth, in contrast to cells that lacked Eras expression. NMuMG cells transduced with ErbB2 alone showed an increased cell proliferation rate, but not under low serum conditions (0.5\%). However, when both Eras and ErbB2 were expressed in these cells, cell proliferation was synergistically increased under all tested conditions. Similar results, including the drastic enhancement of cell proliferation by combined expression of ERAS and ErbB2, were obtained with the human breast epithelial cell line MCF10A, using either murine or human ERAS (Supplementary Fig. 2D). Thus, co-expression of ERAS and ErbB2 synergistically enhances cell proliferation of mammary epithelial cells.

\section{ERAS and ERBB2 collaborate by activating complementary pathways}

To further investigate the observed insulin-independence of cells expressing ERAS and the mechanism of synergism with ErbB2, we grew MCF10A cells with and without these genes in near absence of serum $(0.1 \%)$ and we omitted insulin and EGF from the medium, growth factors that are normally required for growth of these cells (a third factor, hydrocortisone, was always added). Empty vector control cells were unable to grow in absence of either insulin or EGF (Fig. 4a, b), whereas expression of ERAS or ErbB2 provided the cells with the capability to grow in the absence of insulin or EGF, respectively. Co-expression of ERAS and $E r b B 2$ enabled cellular growth in medium depleted from serum, EGF and insulin. EGF primarily functions through the MAPK/ERK pathway via EGFR, whereas insulin 
A

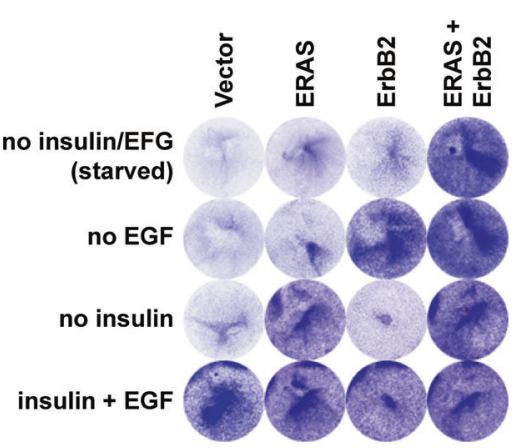

B

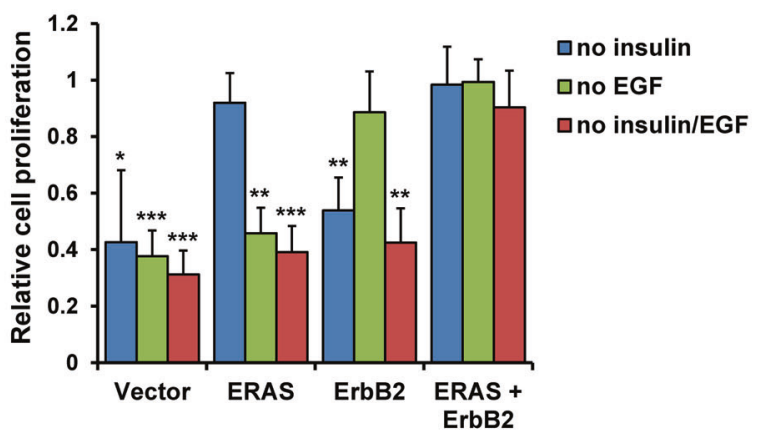

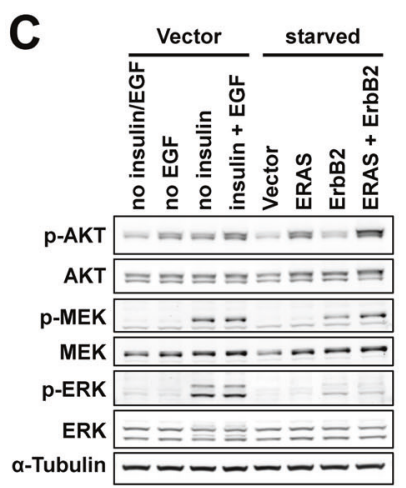

$\mathbf{F}$
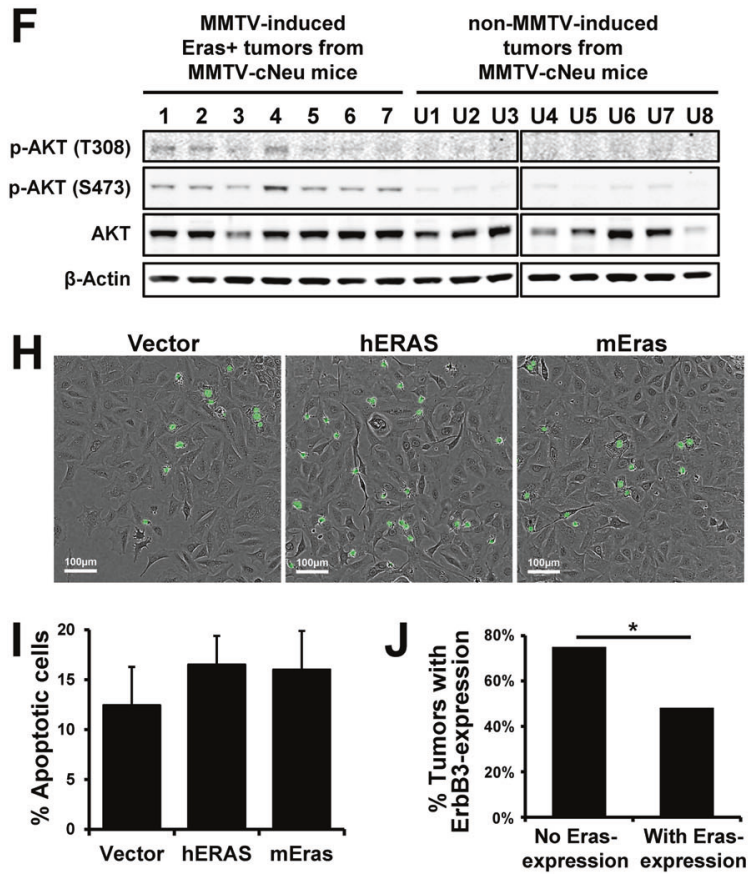
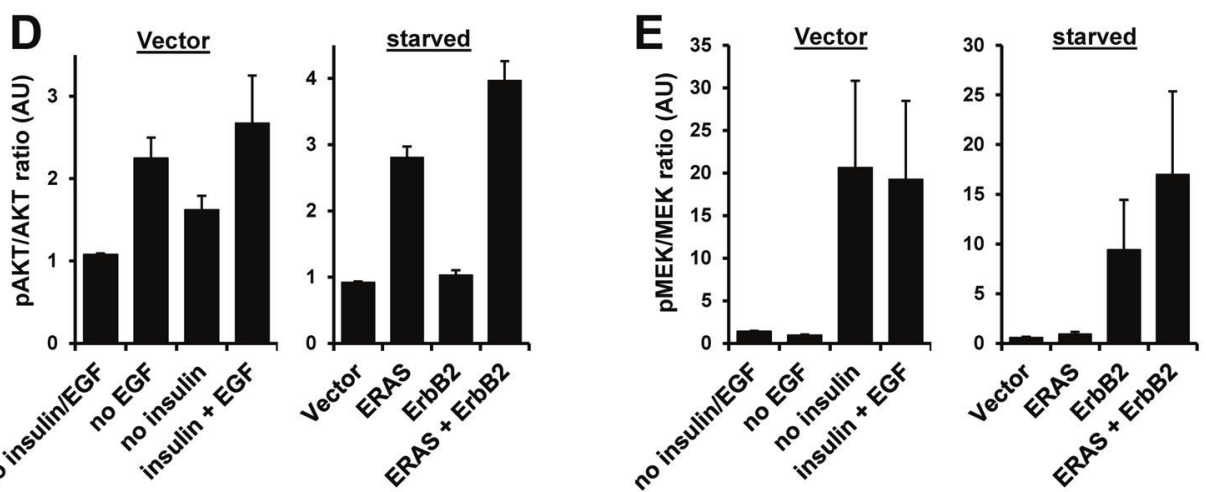

G

MCF10A

NMuMG

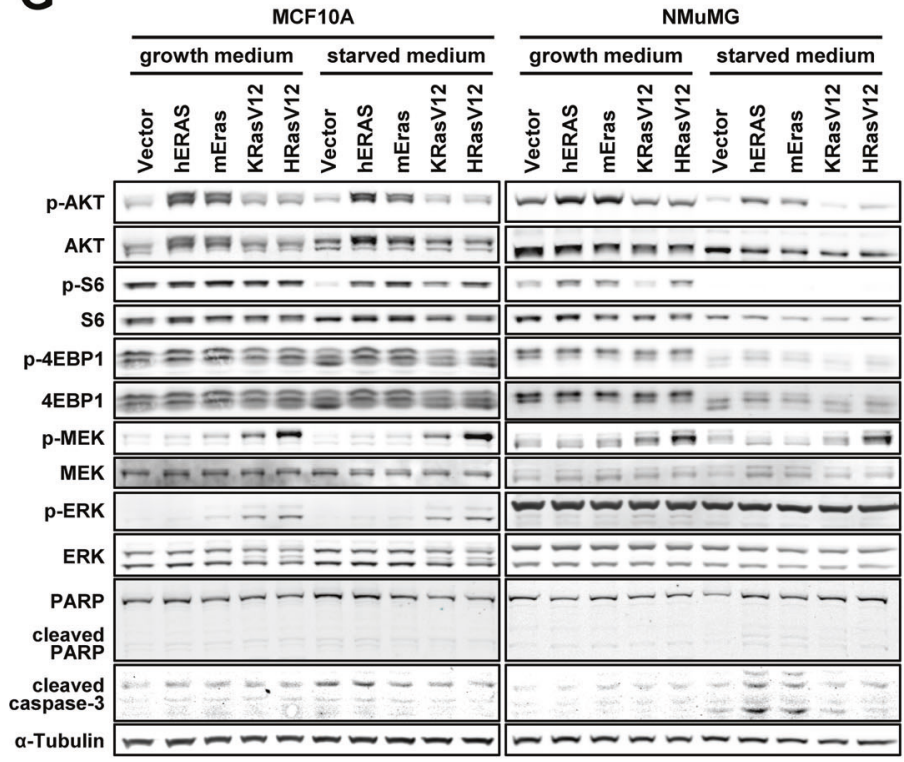

predominantly activates the PI3K/AKT signaling cascade through the insulin receptor (Fig. 4c-e). We therefore hypothesized that ERAS and ERBB2 collaborate through the activation of these complementary pathways. Indeed, it has been shown that ERAS expression activates the PI3K/ AKT pathway in mouse ES cells [21]. Here, we show that also in mammary epithelial cells, expression of ERAS strongly induces PI3K/AKT-pathway activity, as determined by AKT phosphorylation (Fig. 4c, d). Moreover, MMTV-induced Eras expression in primary mammary tumors additionally correlates with PI3K/AKTpathway activation (Fig. 4f). Although ErbB2 expression alone appears to exclusively activate the MAPK/ERK pathway, its co-expression with ERAS additionally enhances PI3K/AKT pathway activity (Fig. 4c, e; Supplementary Fig. 3A). 
Fig. 4 ERAS and ERBB2 activate complementary signaling pathways. a Representative Crystal Violet staining of MCF10A cells, stably transduced with ERAS and ErbB2 alone or co-expressed, compared to vector control cells. 200,000 cells per well (6-wells plates) were allowed to grow for 4 days in medium supplemented with $0.1 \%$ FCS, with and without insulin and EGF (as indicated). b Quantification of cell proliferation in $\mathbf{a}$, relative to the growth condition with both insulin and EGF $(n=4)$, plotted as mean + SD. Significant differences in cell proliferation rates upon omission of growth factors are shown and were determined by a Welch's $t$-test and $p$-values are shown as *p $<0.05$; ** $p<0.01$; *** $p<0.001$. c Representative Western blots showing phosphorylated (p-) and total protein of AKT, MEK, and ERK in MCF10A vector control cells in medium with and without insulin and EGF (as indicated), compared to MCF10A cells in medium devoid of those growth factors (starved), stably transduced with ERAS and $E r b B 2$ alone or in combination (as indicated). All proteins were detected on the same blot and $\alpha$-tubulin was used as loading control. d, e Ratios phosphorylated over total AKT (d) and MEK (e), plotted as mean $+\mathrm{SD}(n=3)$ and quantified from the blots as in c. pERK/ERK ratios are provided in Supplementary Fig. 3A. f Western blots showing phosphorylated (p-)AKT (on the indicated amino acid residues) and total AKT protein in tumors with MMTV induced Eras expression and in non-MMTV induced tumors that arose in ErbB2 transgenic mice. $\beta$ actin was used as loading control. $g$ Western blots showing PI3K/AKT (AKT, S6, 4EBP1), MAPK/ERK (MEK, ERK), and apoptosis (PARP, caspase-3) signaling in MCF10A and NMuMG cells, stably transduced with human or murine ERAS (hERAS or mEras, respectively), KRasV12 or HRasV12, compared to vector control cells. Cells were grown under starved conditions or in complete growth medium. All proteins were detected on the same blot and $\alpha$-tubulin was used as loading control. $\mathbf{h}$ Representative micrographs showing apoptosis in green (fluorescent caspase-3/7 reagent) of 7-day starved MCF10A cells, stably transduced with human or murine ERAS (hERAS or mEras, respectively) and vector control cells (scale bar $=100 \mu \mathrm{m}$ ) (1500 cells per well, 384-wells plates). The same analysis in NMuMG cell is provided in Supplementary Fig. 3B. i Quantification of percentages apoptotic cells of experiment in $\mathbf{h}$, showing mean $+\mathrm{SD}(n=$ $4-7)$. Welch's $t$-test showed no significant differences $(p>0.05)$. The same analysis in NMuMG cell is provided in Supplementary Fig. 3C. j Percentage of ErbB3-expression positive MMTV-induced tumors in tumors with $(n=52)$ or without $(n=27)$ Eras expression, showing a statistically significant difference with $p$-value $=0.0244(*)$, determined by a Fisher's exact test

We explored possible differences in signaling downstream of ERAS compared to other oncogenic RAS-family members. Although ERAS induced potent activation of the PI3K/AKT pathway in mouse mammary cells, it did not activate the MAPK/ERK pathway, in contrast to constitutive active mutants of canonical oncogenic RAS-family members KRAS (KRasV12; Kirsten rat sarcoma viral oncogene homolog) and HRAS (HRasV12; Harvey rat sarcoma viral oncogene homolog) that primarily activated the MAPK/AKT pathway (Fig. 4g: p-AKT, p-ERK, and pMEK).

ERAS could potentially also protect against apoptosis and hence in part explain the observed ERAS-induced cell expansion. However, cells expressing ERAS showed slightly more activation of the apoptosis signaling cascade, which was confirmed microscopically (Fig. 4g-i;
Supplementary Fig. 3B, C). Thus, ERAS does not protect cells from apoptosis, but does strongly induce cell proliferation via PI3K/AKT pathway activation.

ERBB2 forms homo- or heterodimers with other ERBBfamily members, most notably with ERBB3 (erb-b2 receptor tyrosine kinase 3). In this complex, ERBB3 is required for PI3K/AKT pathway signaling and thereby strongly contributes to tumorigenesis [27, 28]. As we established that ERAS is a very potent activator of the PI3K/AKT pathway, we hypothesized that it may functionally replace ERBB3. Indeed, we find significantly less ErbB3-expression among the Eras-positive tumors arising in MMTV-infected ErbB2-transgenic mice, compared to Eras-negative tumors (Fig. 4j). Other MMTV target genes may also be able to replace $\operatorname{ErbB} 3$, as we find that $42 \%$ of MMTV-induced ErbB2-driven tumors lack ErbB3-expression, whereas all spontaneous ErbB2-driven tumors expressed ErbB3 (Supplementary Fig. 3D).

\section{ERAS collaborates synergistically with ERBB2 in tumorigenesis}

To assess whether the collaboration of ERAS with ERBB2 indeed affects tumorigenic potential, we performed a soft agar colony formation assay, as anchorage-independent growth correlates well with in vivo tumorigenicity (Fig. 5a, b; Supplementary Fig. 4A, B). Both in MCF10A and NMuMG cells, expression of ERAS hardly enabled anchorage-independent growth, whereas ErbB2-expression did induce some colony formation. Combined expression of $E R A S$ and $E r b B 2$, however, resulted in a strong synergistic induction of anchorage-independent growth in these cells. Interestingly, this synergism was not observed between ERBB2 and constitutive active KRAS (KRasV12) or HRAS (HRasV12) (Fig. 5b). Hence, the synergistic tumorigenic collaboration with ERBB2 seems unique to ERAS within the RAS family. Moreover, ERAS seems able to functionally replace ERBB3 in induction of anchorage-independent growth in collaboration with ERBB2 (Supplementary Fig. 4C).

To further validate the observed oncogenic synergism between ERAS and ERBB2, we investigated whether combined expression of ERAS and ErbB2 in NMuMG cells indeed synergistically enhances tumor growth in vivo. To this end, we subcutaneously injected $1 \times 10^{6} \mathrm{NMuMG}$ cells transduced with ERAS and ErbB2, alone or in combination, or vector control cells in both flanks of five female BALB/c nude mice and compared tumor latency and growth (Fig. 5c). All mice injected with Eras-transduced cells developed tumors in both flanks, whereas mice injected with the vector control cells did not develop any tumors, even after a prolonged time period, implying that expression of Eras alone is already oncogenic in mammary 
A

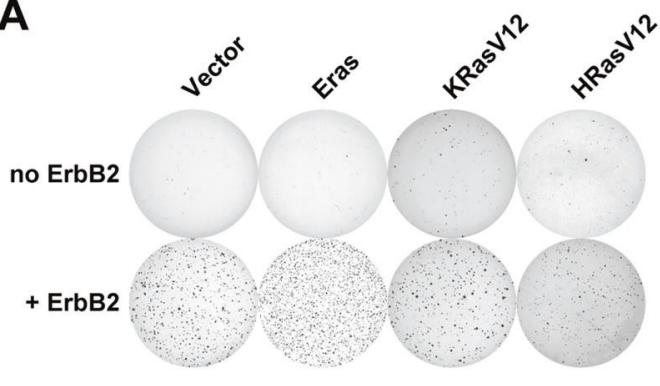

C

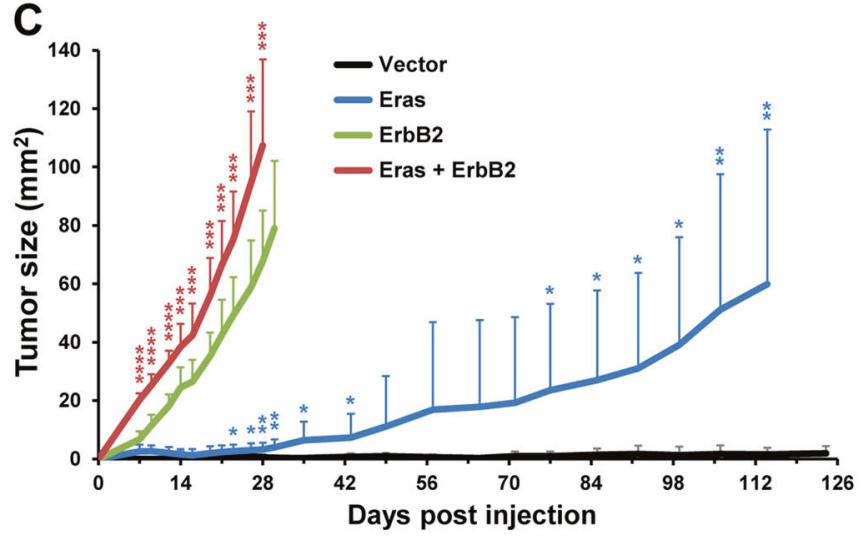

E

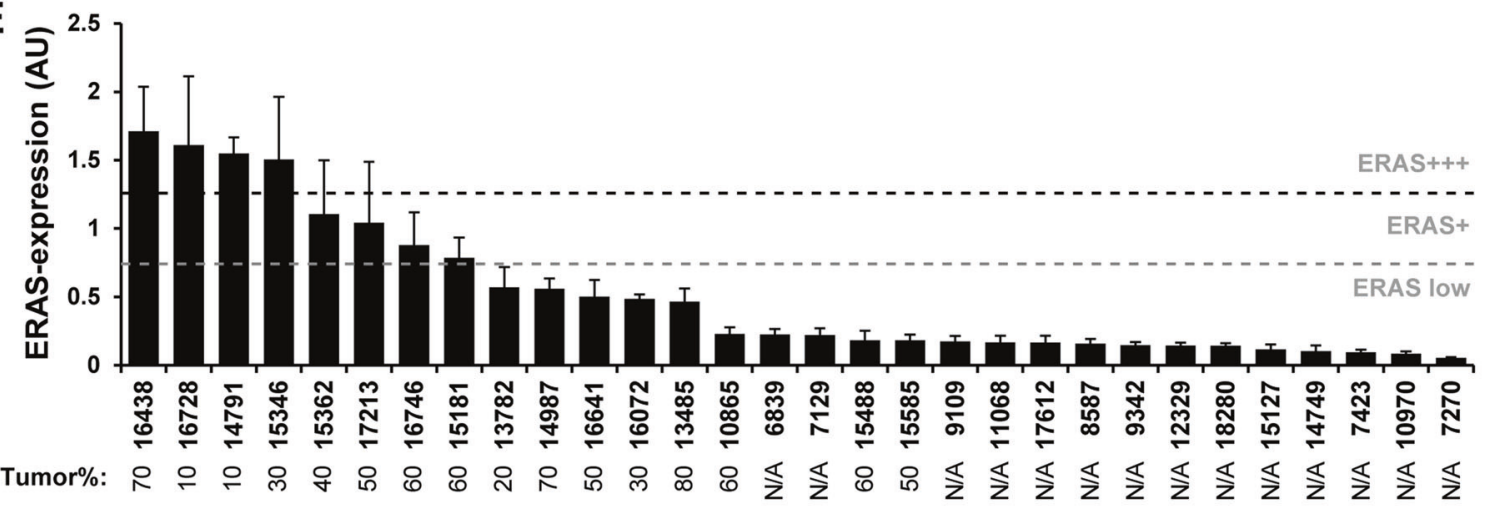

B
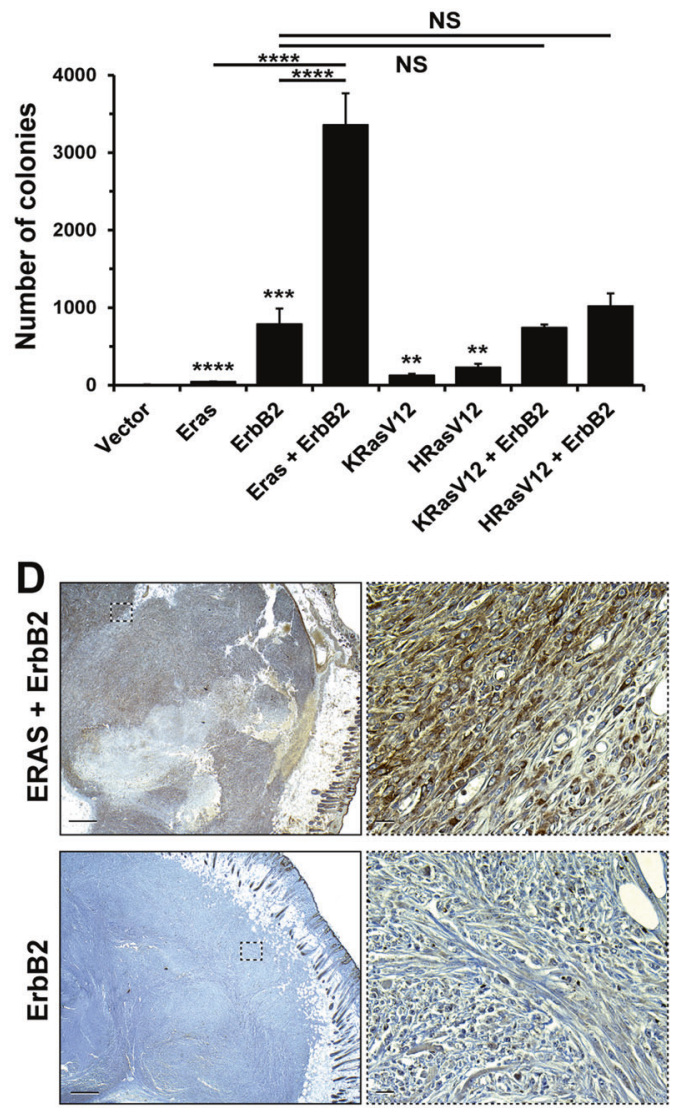

F

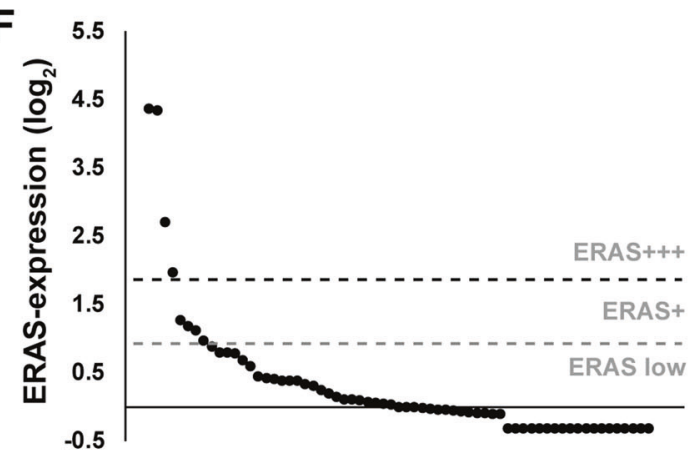

epithelial cells. ErbB2-expressing cells were tumorigenic as expected, but combined with Eras expression, with confirmed ERAS protein expression (Fig. 5d), strongly
G

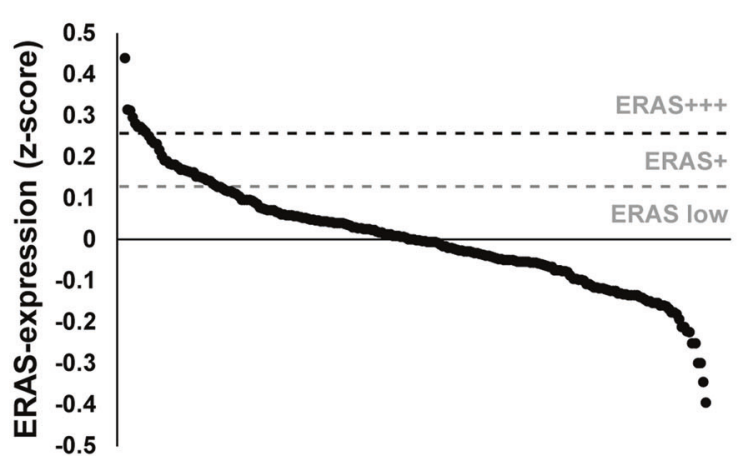

accelerated tumor growth (Fig. 5c), providing in vivo evidence that ERAS and ERBB2 indeed synergistically collaborate in tumorigenesis. 
Fig. 5 ERAS and ERBB2 synergistically induce tumor growth. a Representative whole well scans of NMuMG cells, stably transduced with Eras, KRasV12, or HRasV12 alone or co-expressed with ErbB2, allowed to grow in soft agar for three weeks $(25,000$ cells per well, 6 wells plates). b Quantification of soft agar growth of $\mathbf{a}$, plotted as mean + SD $(n=4-6)$. Statistical significance of differences in relevant comparisons was determined by a Welch's $t$-test and $p$-values are shown as $* p<0.05 ; * * p<0.01 ; * * * p<0.001 ; * * * * p<1 \times 10^{-4}$; NS not significant. Relevant significant differences to the vector control are depicted above the columns. c Tumor growth in mice subcutaneously injected with NMuMG cells ectopically expressing ERAS and $E r b B 2$ alone or in combination, and vector control cells $(n=10$ each). Data are represented as mean + SD. Statistically significant differences in tumor growth between vector controls and Eras (blue) or ErbB2 and Eras + ErbB2 (red) are indicated as $* p<0.05 ; * * p<0.01$; $* * * p<0.001 ; * * * * p<1 \times 10^{-4}$ (Welch's $t$-test). d Representative micrographs of immunohistochemical staining for ERAS (brown) in tumors grown from subcutaneously injected NMuMG cells, stably transduced with ErbB2 with or without Eras, described in c. Right panels are enlargements of the boxed areas. Scale bars: overviews (left) $=500 \mu \mathrm{m}$, details (right) $=20 \mu \mathrm{m}$. e Quantitative RT-PCR of ERAS mRNA expression in 30 HER2-positive primary breast carcinomas. Lower thresholds for ERAS positive (ERAS+; above median $+\mathrm{SD})$ and highly positive (ERAS +++ ; above median $+2 \times \mathrm{SD})$ are indicated with a gray and black dashed line, respectively. The estimated tumor percentages are indicated below each sample: N/A, not available. f, g Median-centered ERAS mRNA expression levels in HER2-positive tumor samples (based on PAM50 gene signature) derived from The Cancer Genome Atlas (TCGA) RNA-seq data $(n=$ 65) [29] (f) or METABRIC microarray data $(n=220)$ [30] (g). Thresholds for ERAS positive (ERAS+) and highly positive (ERAS+ ++ ) expression levels are indicated as in $\mathbf{e}$

\section{ERAS is expressed in a subset of human primary breast carcinomas}

To determine the clinical significance of ERAS, we used RT-PCR to examine the expression of ERAS mRNA in a large group of primary human breast carcinomas from patients treated in our institute (Supplementary Fig. 4D; Supplementary Table 1). Five out of 51 tumors (10\%) showed clear ERAS expression, confirmed by immunohistochemistry (Supplementary Fig. 4E), and an additional nine breast cancer samples exhibited lower levels of ERAS mRNA. Sequencing of the PCR-products excised from the gel confirmed that the amplified cDNA represented ERAS mRNA of human origin. Surprisingly, only few of the ERAS-positive tumors were also categorized as HER2+ (Supplementary Table 1), where we would expect an enrichment of ERAS positive tumors, based on our in vitro and in vivo studies. We therefore tested an independent uniform HER2+ human breast cancer series for ERAS expression by quantitative RT-PCR (Fig. 5e). Eight out of the 30 tested HER2+ tumors (27\%) could be scored as ERAS positive, of which four cases (13\%) were highly positive. This may even be an underestimation due to the low tumor percentage in some of the samples (Fig. 5e). We did not observe samples with both high expression of ERAS and ERBB3 (Supplementary Fig. 4F, G), strengthening our notion that ERAS may functionally replace ERBB3. The publicly available expression datasets of The Cancer Genome Atlas (TCGA) (RNA-seq) [29] and METABRIC (microarray) [30] both confirm ERAS expression in a subset of human HER2+ breast cancers, albeit with somewhat lower percentages (TCGA: 12\% ERAS +, 6\% ERAS +++; METABRIC: $16 \%$ ERAS,$+ 7 \%$ ERAS +++ ) (Fig. 5f, g). These results point at a role for this intrinsically active and oncogenic RAS-like gene in human breast cancer.

\section{ERAS induces resistance to HER2-targeting therapy}

Although HER2-targeting therapy has improved clinical outcome, therapy resistance remains a considerable problem in the clinic. Previously, PI3K/AKT pathway hyperactivation has been associated with therapy resistance in HER2+ breast cancer [31-33]. As our data show that ERAS constitutively activates this pathway, we interrogated whether expression of ERAS could also alter the response to HER2targeting therapy. Indeed, we found that $E R A S$ expression in the HER2+ breast cancer cell lines SKBR3 and BT474, leads to a marked reduction in sensitivity to both the ERBB2-targeting monoclonal antibody Trastuzumab and the small molecule ERBB2-kinase inhibitor Lapatinib, enabling sustained tumor cell growth (Fig. 6a-c; Supplementary Fig. 5A, B).

To confirm that the mechanism of therapy resistance by ERAS is due to its potent PI3K/AKT pathway activation, we treated HER2+ breast cancer cell lines with suboptimal concentrations of Trastuzumab or Lapatinib, in combination with the PI3K inhibitor GDC-0941 (Fig. 6d, e; Supplementary Fig. 5C-E). Although single-agent treatments had little to no influence on the proliferation rate of breast cancer cells expression both ERBB2 and ERAS, the combination of HER2- and PI3K-targeting agents potently reduced cell proliferation. These data strongly suggest that PI3K/AKT-pathway activation by ERAS confers resistance to HER2-targeting therapeutics. Moreover, these data further support our conclusion that the observed synergism between ERBB2 and ERAS in tumorigenesis is due to their activation of the complementing oncogenic MAPK/ERK and PI3K/AKT pathways, respectively.

To validate ERAS-induced resistance in vivo, we subcutaneously injected $1 \times 10^{6}$ NMuMG cells expressing ErbB2, alone or in combination with Eras, in opposite flanks of 18 female BALB/c nude mice. When tumors had been established in both flanks, we randomly assigned nine mice to receive Lapatinib treatment (Fig. 6f, g). Tumor growth was significantly reduced upon Lapatinib treatment in flanks injected with cells only expressing ErbB2. In contrast, tumors expressing both ErbB2 and Eras showed 
A

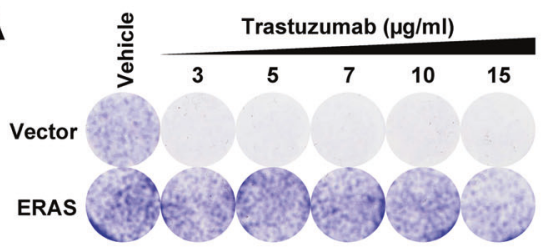

B

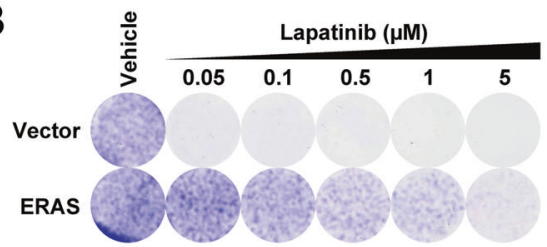

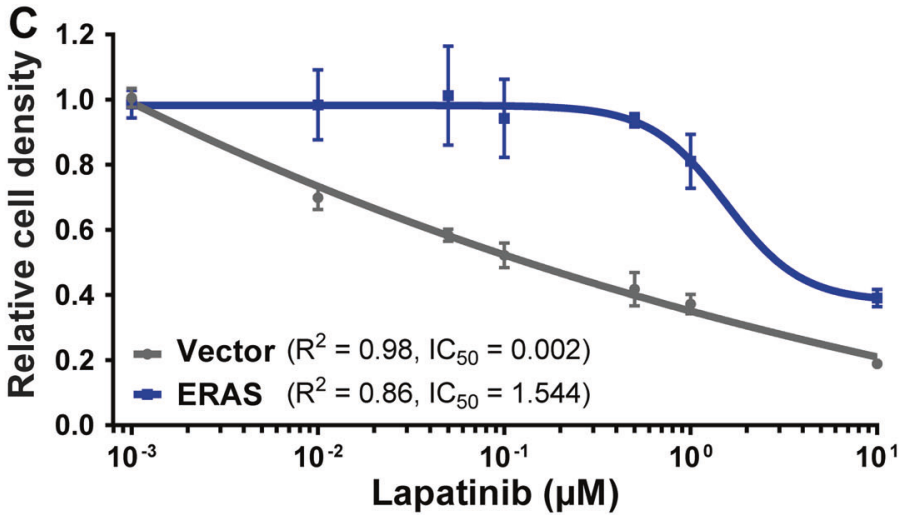

E
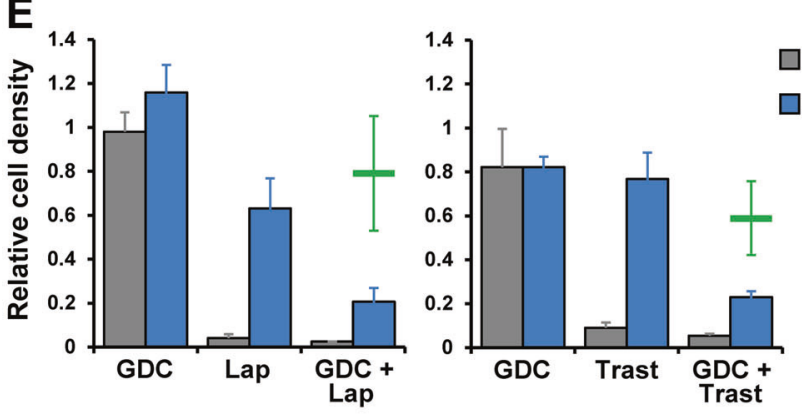

$\mathbf{F}$
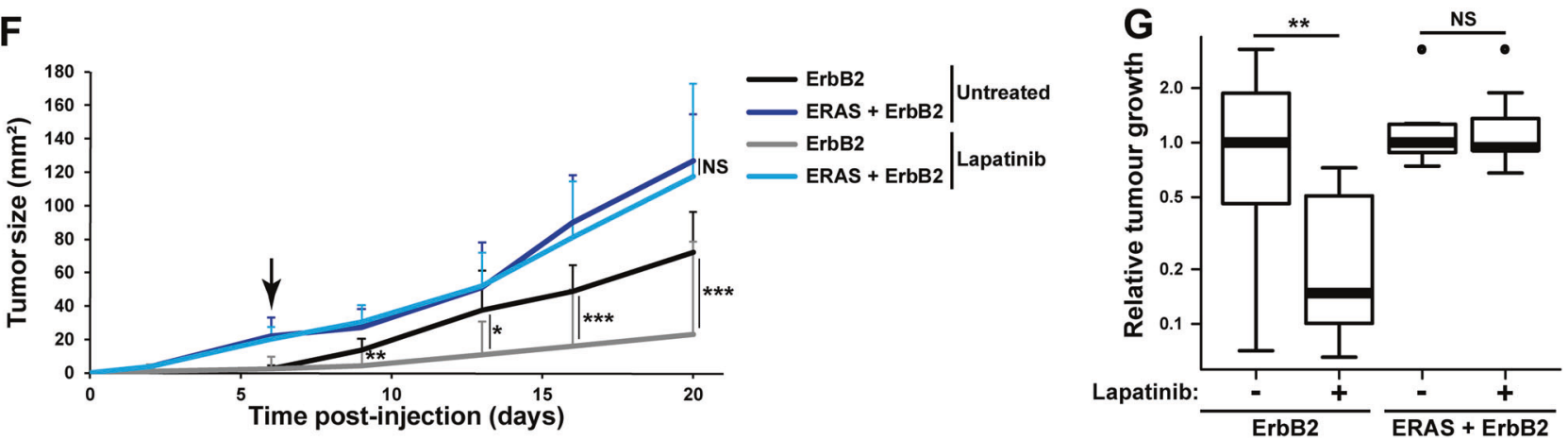

Fig. 6 ERAS induces Trastuzumab and Lapatinib resistance via PI3K/ AKT-pathway hyperactivation. a, b Crystal Violet staining of BT474 vector controls or BT474 cells stably transduced with ERAS, allowed to grow for 7 or 10 days in medium supplemented with the indicated concentrations Trastuzumab (a) or Lapatinib (b), respectively $(25,000$ cells per well, 24-wells plates). c Lapatinib dose response curves of SKBR3, stably transduced with ERAS, or vector control cells. The data points represent the mean $\pm \mathrm{SD}(n=4)$ of the cell density of the Lapatinib-treated to vehicle control cells (initially, 1000 cells per well were plated, 384-wells plate). The fitted curve and associated $R^{2}$ and $\mathrm{IC}_{50}$ values are shown. d Representative Crystal Violet staining of BT474 vector controls or BT474 cells stably transduced with ERAS, grown in presence of $20 \mathrm{nM}$ Lapatinib (grown 13 days), $5 \mu \mathrm{g} / \mathrm{ml}$ Trastuzumab (grown 11 days) and/or $20 \mathrm{nM}$ PI3K inhibitor GDC0941 , or equal volumes of DMSO (vehicle) $(50,000$ cells per well, $12-$

no response to Lapatinib, confirming ERAS-induced Lapatinib resistance in vivo.

In analogy to acquired therapy resistance in patients with HER2 + breast cancer, we investigated whether expression wells plates). (e) Quantification of (d), showing cell proliferation relative to vehicle controls. Data plotted as mean $+\mathrm{SD}(n=3)$. A projection of the combined (i.e., additive) single-agent effects of GDC0941 (GDC) and Lapatinib (Lap) or Trastuzumab (Trast) is shown in green. f Tumor growth of NMuMG cells, stably transduced with $E r b B 2$ alone or together with ERAS, subcutaneously injected in opposite flanks of BALB/c nude mice, untreated or treated with 100 $\mathrm{mg} / \mathrm{kg} /$ day Lapatinib ( $n=9$ each). When tumors were established in both flanks, mice were randomly allocated to either group before treatment was started (indicated by arrow). Significant differences in growth rate between treated and untreated tumors are determined by a Welch's $t$-test: $* p<0.05$; $* * p<0.01 ; * * * p<0.001$; NS not significant. $\mathbf{g}$ Boxplot of tumor growth from start of treatment to day 20 of experiment in $\mathbf{f}$, relative to growth of untreated controls. Statistical analysis was performed by a Welch's $t$-test: $*^{*} p<0.01$; NS not significant

of endogenous ERAS could also be attained in treatmentnaive HER2 + breast cancer cell lines under selective pressure of Trastuzumab or Lapatinib. To this end, we cultured SKBR3 and BT474 cells in medium containing 

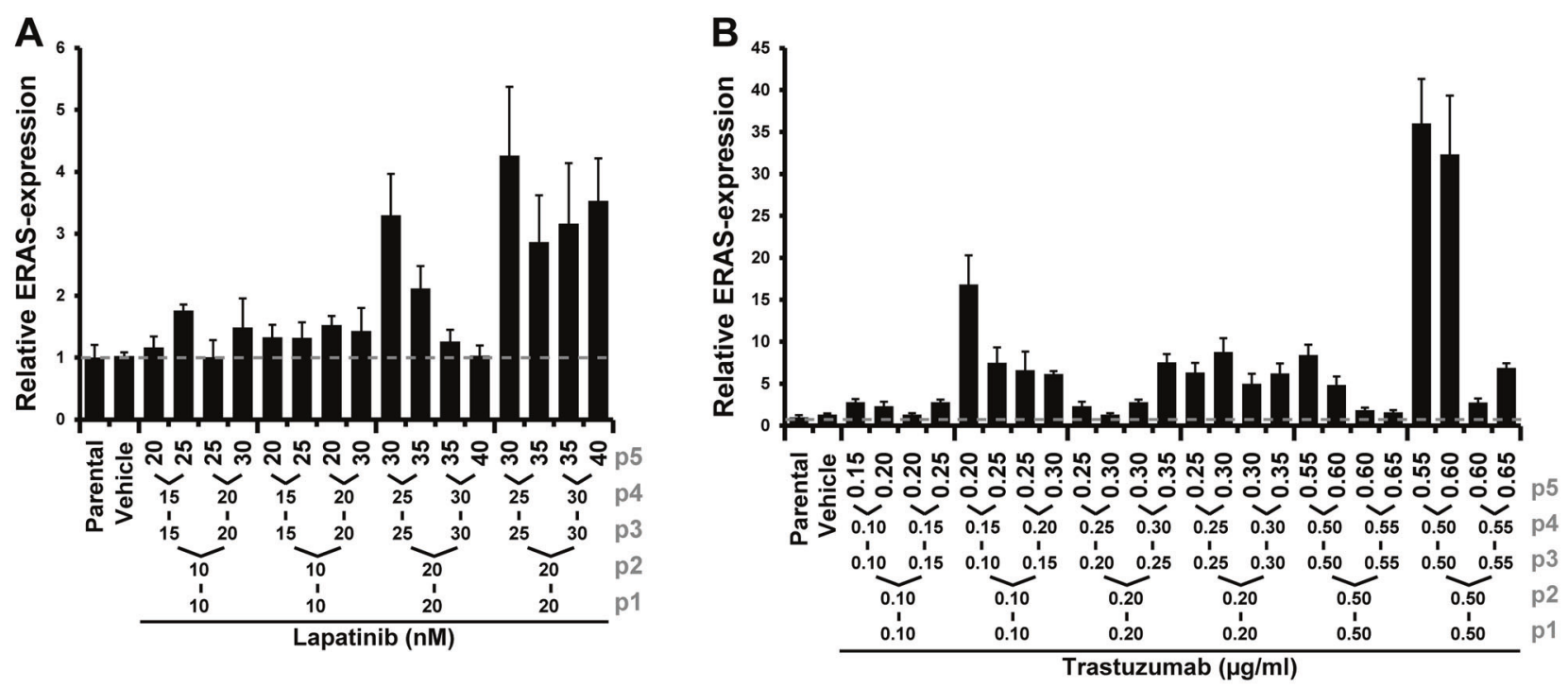
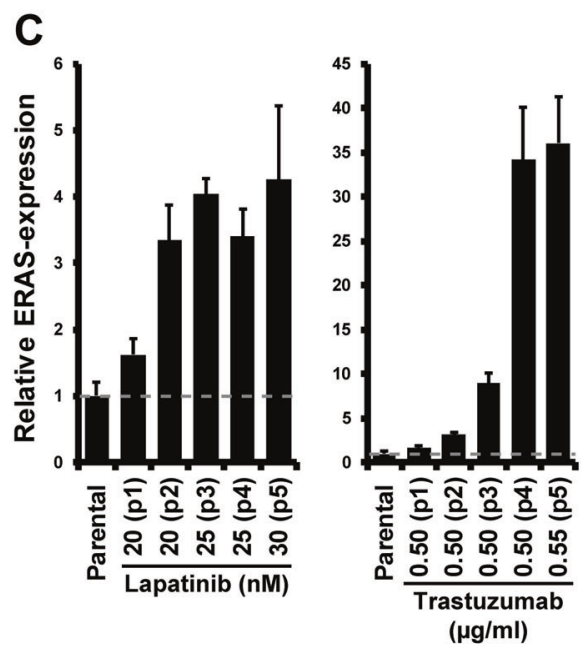

D
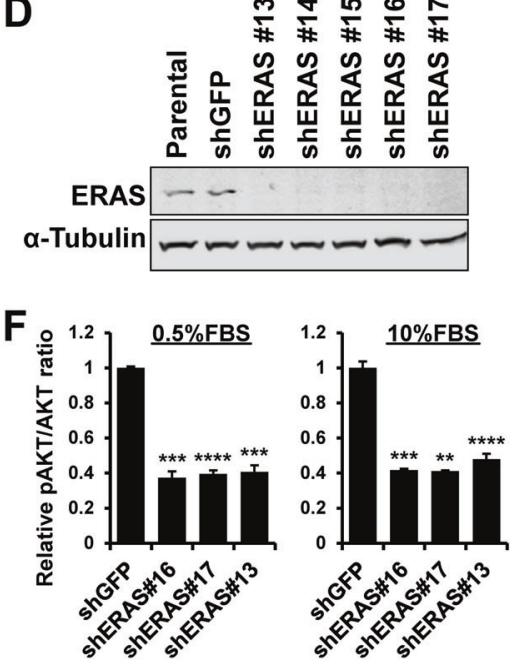

E
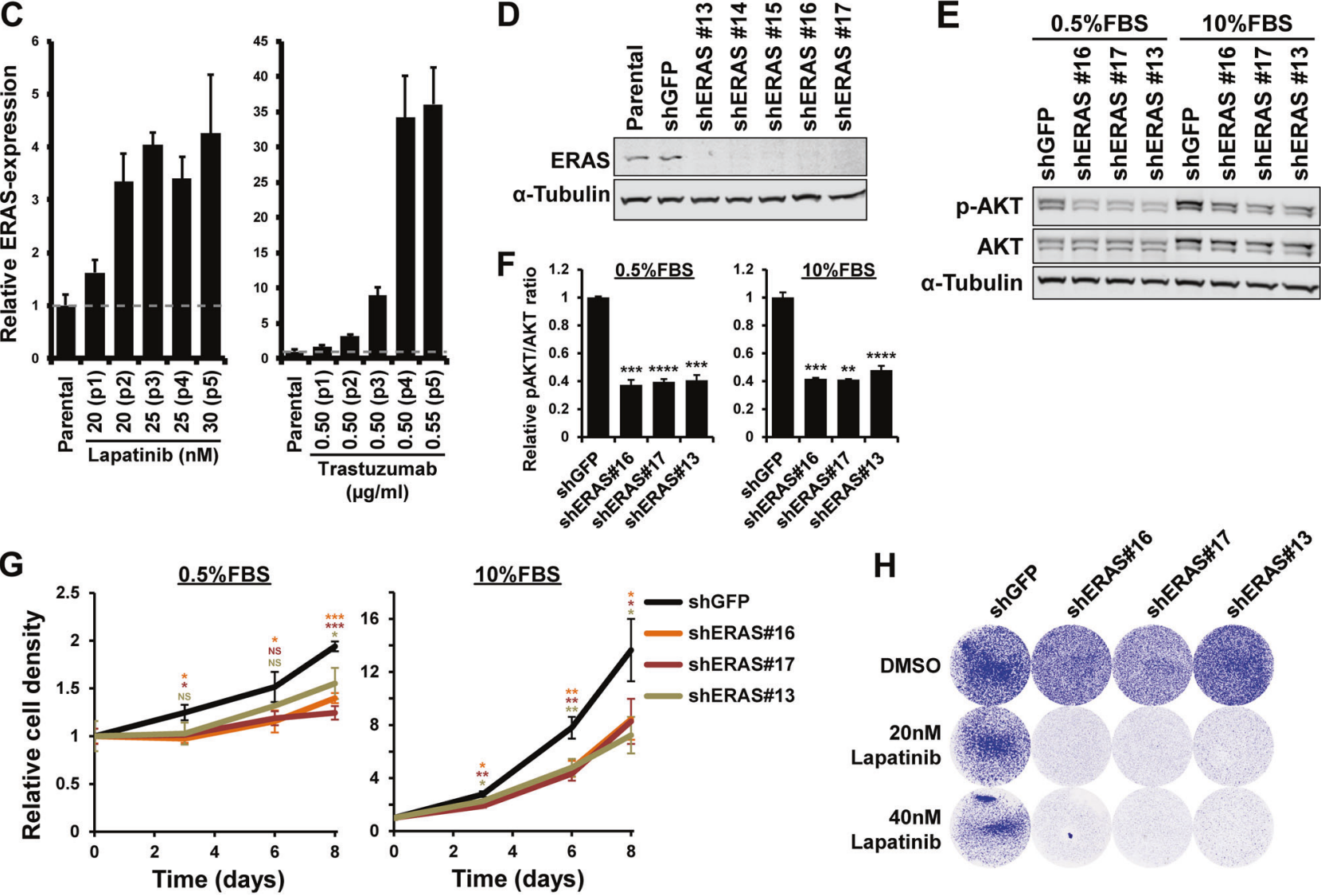

H

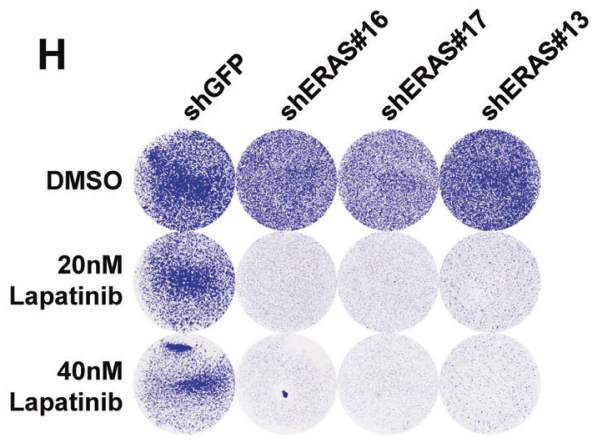

increasing concentrations of Trastuzumab or Lapatinib. In more than half of the cultures, ERAS expression was induced within five passages in the presence of Trastuzumab or Lapatinib, especially at higher drug concentrations (Fig. 7a, b; Supplementary Fig. 6A, B). Detailed expression analysis of each passage revealed that ERAS expression increases gradually with every passage, indicating a selection for increasing ERAS expression by the increasing drug concentrations (Fig. 7c; Supplementary Fig. 6C). In contrast, untreated control cells did not show increased ERAS expression after five passages. Subsequent knockdown of Trastuzumab-induced endogenous ERAS expression in BT474 and SKBR3 cultures with the highest induction (designated: BT474/ $\mathrm{R}^{\mathrm{ERAS}+}$ and SKBR3/R ${ }^{\mathrm{ERAS}+}$, respectively) resulted in a concomitant significant reduction in PI3K/AKT-pathway activation, cell proliferation and 
Fig. 7 HER2-targeted treatment selects for cells expressing ERAS. a, b ERAS-mRNA expression levels in BT474 cells (parental), cultured for five passages in presence of vehicle (to aid interpretation of the graph this level is also indicated by a gray dashed line) or the indicated concentrations Lapatinib (a) or Trastuzumab (b). Data are represented as mean $+\mathrm{SD}$ of triplicates and are shown relative to ERAS levels in vehicle controls. Lapatinib or Trastuzumab concentrations after each passage are shown below and passage number $(\mathrm{p})$ is depicted in gray at the right. The same experiment using SKBR3 cells is provided in Supplementary Fig. 6A and B. c Relative ERAS-expression levels at each passage in the cultures that acquired the highest ERAS levels under Lapatinib or Trastuzumab selection depicted in $\mathbf{a}$ and $\mathbf{b}$, respectively. d Western blot, showing ERAS knockdown efficiency in the Trastuzumab-treated BT474 cell culture of c (designated: BT474/ $\mathrm{R}^{\text {ERAS+ }}$ ). $\alpha$-Tubulin was used as loading control. The same analysis in SKBR3 cells is provided in Supplementary Fig. 6C. e Representative Western blots showing phosphorylated (p-) and total protein of AKT in a selection of the cell cultures from $\mathbf{d}$. $\alpha$-Tubulin was used as loading control. f Ratios phosphorylated over total AKT, relative to shGFP controls, quantified from the blots as in e. Data represented as mean $+\mathrm{SD}(n=3)$ and statistically significant differences in PI3K/ AKT-pathway activation between shGFP controls and shERAS are indicated as ${ }^{* *} p<0.01 ; * * * p<0.001 ; * * * * p<1 \times 10^{-4}$ (Welch's $t$ test). The same experiment using SKBR3 cells is provided in Supplementary Fig. 6G. g Growth curves of the BT474/R $\mathrm{R}^{\mathrm{ERAS}+}$ cell culture as in $\mathbf{f}$ in medium supplemented with $10 \%$ FBS or $0.5 \%$ FBS, based on Crystal Violet staining (50,000 cells per well, 24-wells plates). Plotted values are mean $\pm \mathrm{SD}$, relative to day $0(n=3)$. Statistical significance of differences in cell proliferation between shGFP controls and shERAS was determined by a Welch's $t$-test and $p$-values are shown as $* p<0.05$; * $p<0.01$; *** $p<0.001$; NS not significant. The same experiment using SKBR3 cells is provided in Supplementary Fig. $6 \mathrm{H}$. h Representative Crystal Violet staining $(n=3)$ of the BT474/R $\mathrm{R}^{\mathrm{ERAS}+}$ cells as in $\mathbf{f}$, allowed to grow for 6 days in the presence of $20 \mathrm{nM}$ or $40 \mathrm{nM}$ Lapatinib or equal volumes of vehicle (DMSO) (50,000 cells per well, 24-wells plates). The same experiment using SKBR3 cells is provided in Supplementary Fig. 6I

Lapatinib resistance (Fig. 7d-h; Supplementary Fig. 6D-I). These data further prove that induction of ERAS expression renders tumor cells insensitive to HER2-targeted therapy and as such present a new mechanism of therapy resistance.

\section{Discussion}

To shed more light on HER2 + breast tumorigenesis and the mechanisms underlying resistance to HER2-targeting therapeutics, we performed high-throughput MMTV-mediated insertional mutagenesis screens in an MMTV-cNeu transgenic mouse model for HER2+ human breast cancer to discover genes collaborating with ERBB2. Here, we identify several novel candidate oncogenes and assess their correlation with oncogenic ErbB2 (HER2/neu). We show that loci containing $W n t$ and $F g f / F g f r$-family genes have a significant anti-correlation with the ErbB2-transgene, whereas the Lrfn5 and Gm26870/Mir101c loci, but especially the Eras locus, are preferentially targeted by MMTV in the ErbB2-transgenic mice. The candidate target LRFN5 (leucine-rich repeat and fibronectin type III domain- containing 5; also known as SALM5, Synaptic AdhesionLike Molecule 5) is a poorly studied transmembrane adhesion molecule, involved in synapse formation and neurite outgrowth $[34,35]$. Of the candidates in the other ErbB2-associated MMTV-targeted locus, the long intergenic non-coding RNA $G m 26870$ has never been described, to our knowledge, whereas microRNA-101c (mmи-mir101c) has been implicated in cancer before [36]. Although it may be worthwhile to investigate these CISs in the context of HER2 + breast cancer, we focused on Eras in this study.

Eras is a unique RAS-family member in various aspects. The gene encodes a constitutively active small GTPase and appears to be exclusively present in mammalian genomes [37]. In mice, Eras has been reported to be expressed only in undifferentiated ES cells and teratocarcinomas derived from these cells [21], whereas in human ES cells, ERAS expression has not been detected [38]. In mouse ES cells, Eras has been shown to activate the PI3K/AKT pathway but not the MAPK/ERK pathway [21], which we also observe in both murine and human mammary cells upon ectopic expression. This is in contrast to the canonical RASfamily members, KRAS, HRAS, and NRAS, which primarily activate the MAPK/ERK pathway instead. ERAS is not required for pluripotency of ES cells, but growth rate and tumorigenicity of ERAS-null ES cells is significantly reduced, which can be rescued by expression of activated PI3K. This has led to the conclusion that ERAS-induced PI3K/AKT-pathway activation is important for the tumorlike growth capacity of ES cells. In accordance, ectopic expression of Eras also confers tumorigenic properties to murine embryonic NIH-3T3 fibroblasts [21] and ERAS expression has been associated with human gastric cancer $[39,40]$. In this study, we show that Eras is not expressed during any developmental stage of the adult mouse mammary gland, but that the gene acts as a tumorigenic driver upon ectopic expression in mammary epithelial cells. We specifically demonstrate that Eras induces increased cell proliferation and insulin-independent growth.

Importantly, we show that ERAS and ERBB2 collaborate to synergistically enhance cell proliferation, anchorageindependent growth in vitro and tumorigenesis in vivo. In human and mouse mammary tumors, ERBB2 is usually present in a heterodimer with ERBB3 [41], in which ERBB2 activates the MAPK/ERK pathway, whereas ERBB3 activates the PI3K/AKT pathway. We show that $42 \%$ of ErbB2-driven tumors with CIS-associated MMTV insertions no longer express ErbB3, in contrast to the spontaneous tumors in non-infected $E r b B 2$-transgenic mice, which express $E r b B 3$ in $100 \%$ of the cases, suggesting that the activated MMTV-targets functionally replace ERBB3. Indeed, in its capacity to constitutively activate the PI3K/ AKT pathway, the MMTV target Eras is able to adopt this role from ERBB3. Correspondingly, tumors expressing 
both $E R A S$ and $E R B B 3$ are rare or absent in both the human and murine setting.

Only half of HER2+ breast cancer cases respond to HER2-targeted therapies and this therapy resistance is also reflected in ErbB2-transgenic mouse models [42, 43]. Notably, PI3K/AKT-pathway activation is implicated in resistance to the commonly used HER2-targeted therapeutics, Trastuzumab and Lapatinib, specifically through activating mutations in PIK3CA (phosphatidylinositol-4,5bisphosphate 3-kinase, catalytic subunit alpha) or loss of its antagonist PTEN (phosphatase and tensin homolog) [31-33, 44-48]. Our data identify ERAS as an important gene that hyperactivates the PI3K/AKT pathway and induces resistance to HER2-targeting therapeutics, both in vitro and in vivo. Moreover, we found that ERAS mRNA is expressed in at least $13 \%$ of HER2 + human breast carcinomas, further subscribing the clinical relevance of ERAS. Altogether, these results suggest that ERAS expression may be a promising biomarker for de novo therapy resistance. We also demonstrate that ERAS may be involved in acquired resistance, as selective pressure by Trastuzumab and Lapatinib leads to upregulation of ERAS expression in growing HER2+ breast cancer cells.

The standard treatment for HER2+ breast carcinomas comprises the combination of chemotherapy with Trastuzumab, followed by Lapatinib treatment when tumors do not respond to Trastuzumab. Considering that ERAS was recently also implicated in resistance to chemotherapeutic agents in neuroblastoma and gastric cancer cell lines [49, 50], the clinical importance of this gene for therapeutic response is further highlighted. However, large-scale validation of ERAS expression as predictive marker for therapy response is hampered by the lack of an ERAS probe on the gene expression microarrays used in clinical trials. Promisingly though, we observed in a pre-clinical setting that the combination of suboptimal doses of a PI3-kinase inhibitor with suboptimal doses of Trastuzumab or Lapatinib can abrogate ERAS-induced therapy resistance and the oncogenic synergism between ERAS and ERBB2. This combination therapy may therefore offer an important window of opportunity for treatment of HER2+ breast cancer patients with primary or acquired resistance to HER2-targeting drugs.

\section{Material and methods}

\section{Patient samples}

Our pathology department provided 86 random human primary breast carcinoma samples and associated clinical parameters from women treated between 2007 and 2011 at the NKI. In addition, the NKI's Core Facility Molecular
Pathology \& Biobanking (CFMPB) supplied us with RNA samples of 30 randomly selected tumors from a uniform set of 129 HER 2 + primary breast carcinomas, obtained from patients treated between 1989 and 2006 at our institute. All patients individually approved the use of tumor tissue for research purposes via opt-out and specific use for this study was approved by the local Translational Research Board, following positive recommendation of the Medical Ethical Committee.

\section{Insertional mutagenesis screens and tumorigenicity assays}

The MMTV-induced insertional mutagenesis screens and insertion site mapping followed the previously reported classical splinkerette PCR method [11] or the "ShearSplink" method [14]. MMTV-cNeu mice (FVB/N-Tg (MMTVneu)202Mul/J, MGI ID: 1930204), were originally generated by William Muller [7] and bred at the NKI. Tumorigenicity following subcutaneous injection of $1 \times 10^{6}$ viable cells in both flanks of 4-week-old female $B A L B /$ cABomA-nu/nu (BALB/c nude) mice ( $n=10$ for each group), bred in our institute, was assessed as previously described [15]. The in vivo Lapatinib-resistance experiment and analysis was performed in 4-week-old female BALB/ cAnNRj-Foxn $^{n u}$ mice (Janvier Labs, Le Genest-Saint-Isle, France) ( $n=9$ in each group) as described before [15]. Sample size was chosen based on previous experience in $\mathrm{s}$. c. tumor growth studies [15]. No animals were excluded in these studies, except animals that died prematurely because of causes unrelated to the experiment. All grafted animals within the experiment were culled when the largest tumors reached $\sim 1.5 \mathrm{~cm}$ or the animals reached the age of 4 months. The observer was blinded to the type of treatment when measuring tumor size. The "survival" package in the statistical programming language $\mathrm{R}$ was used to plot the Kaplan-Meier curves and to calculate log-rank tests. All mouse experiments were approved by the local Animal Experiments Committee (DEC nr 08.061) and strictly followed the Dutch Code of Practice for Research with Laboratory Animals in Cancer Research.

\section{Cell culture, vectors, and in vitro experiments}

Details on the sources of the used cell lines, culturing conditions, transduction, in vitro treatments and cell proliferation, apoptosis, dose response, and anchorageindependent growth (soft agar) assays are described before [15]. Constructs containing full-length human and murine ERAS-cDNA were a kind gift from Liesbeth Vredeveld (NKI, Amsterdam, The Netherlands). These genes were cloned into pBABE-plasmids with the puromycin resistance gene, using the restriction sites of BamHI and 
SalI. pcDNA3-NeuNT construct was kindly provided by Bill Muller (McGill Cancer Center, Montreal, Canada) and the rat $c N e u(E r b B 2)$-cDNA from this construct was cloned into a pMSCV-plasmid carrying the blasticidin resistance marker, using HindIII and EcoRI restriction sites. pBABEvectors containing human RasV12 and KRasV12 were obtained from the labs of Daniel Peeper and René Bernhards (NKI, Amsterdam, The Netherlands), respectively. Empty backbones of these vectors were used as controls.

\section{Expression analysis, Western blotting, and immunohistochemistry}

RNA isolation from tissues, tumors, and cell lines, followed by RT-PCR and expression analysis was performed as previously described [15]. Primer sequences are provided in the Supplementary Table 3. Details on lysis and subsequent Western blotting are provided elsewhere [15]. Antibody sources and dilutions used can be found in the Supplementary Table 4 . Immunohistochemistry was performed on $4 \mu \mathrm{m}$ sections of paraffin-embedded EAF-fixed tumors using a 1:600 diluted polyclonal antibody targeting ERAS (\#AP1470; Abgent, San Diego, CA, USA). Before staining the sections were subjected to heat-induced antigen retrieval using a pH 6.1 citrate buffer. The specificity of the antibody is provided by the supplier and was further validated in our own lab (Supplementary Table 4)

\section{Statistical analyses}

The number of biological replicates of the tests are provided in the legend of the figures $(n \geq 3)$. The statistical programming language $\mathrm{R}$, including the "Bioconductor" and "Survival" packages, were used for statistical analyses. All the statistical tests are specified in the text and/or figure legends. A $p$-value $<0.05$ was considered statistically significant. Distributions and variances of samples (test and control groups) were similar, as determined using R.

Acknowledgements We are grateful to Hugo Horlings and Sabine Linn for support with acquiring clinical samples. We thank William Muller, Joyce Taylor-Papadimitriou, Liesbeth Vredeveld, Katrien Berns, Daniel Peeper, and René Bernhards for sharing materials. We acknowledge graduate students Sarah-Jane Remak and Nina Borràs Agustí for technical assistance.

\section{Compliance with ethical standards}

Conflict of interest The authors declare that they have no competing interests.

Open Access This article is licensed under a Creative Commons Attribution-NonCommercial-ShareAlike 4.0 International License, which permits any non-commercial use, sharing, adaptation, distribution and reproduction in any medium or format, as long as you give appropriate credit to the original author(s) and the source, provide a link to the Creative Commons license, and indicate if changes were made. If you remix, transform, or build upon this article or a part thereof, you must distribute your contributions under the same license as the original. The images or other third party material in this article are included in the article's Creative Commons license, unless indicated otherwise in a credit line to the material. If material is not included in the article's Creative Commons license and your intended use is not permitted by statutory regulation or exceeds the permitted use, you will need to obtain permission directly from the copyright holder. To view a copy of this license, visit http://creativecommons. org/licenses/by-nc-sa/4.0/.

\section{References}

1. Engstrøm MJ, Opdahl S, Hagen AI, Romundstad PR, Akslen LA, Haugen OA, et al. Molecular subtypes, histopathological grade and survival in a historic cohort of breast cancer patients. Breast Cancer Res Treat. 2013;140:1-11.

2. Dawood S, Broglio K, Buzdar AU, Hortobagyi GN, Giordano SH. Prognosis of women with metastatic breast cancer by HER2 status and trastuzumab treatment: an institutional-based review. J Clin Oncol 2010;28:92-8.

3. Blackwell KL, Burstein HJ, Storniolo AM, Rugo H, Sledge G, Koehler M, et al. Randomized study of lapatinib alone or in combination with trastuzumab in women with ErbB2-positive, trastuzumab-refractory metastatic breast cancer. J Clin Oncol. 2010;28:1124-30.

4. Gagliato DDM, Jardim DLF, Marchesi MSP, Hortobagyi GN, de Melo Gagliato D, Leonardo Fontes Jardim D, et al. Mechanisms of resistance and sensitivity to anti-HER2 therapies in HER2+ breast cancer. Oncotarget. 2016;5:64431-46.

5. Muller WJ, Sinn E, Pattengale PK, Wallace R, Leder P, Singlestep induction of mammary adenocarcinoma in transgenic mice bearing the activated c-neu oncogene. Cell. 1988;54:105-15.

6. Bouchard L, Lamarre L, Tremblay PJ, Jolicoeur P. Stochastic appearance of mammary tumors in transgenic mice carrying the MMTV/c-neu oncogene. Cell. 1989;57:931-6.

7. Guy CT, Webster Ma, Schaller M, Parsons TJ, Cardiff RD, Muller WJ. Expression of the neu protooncogene in the mammary epithelium of transgenic mice induces metastatic disease. Proc Natl Acad Sci Usa 1992;89:10578-82.

8. Andrechek ER, Hardy WR, Siegel PM, Rudnicki MA, Cardiff $\mathrm{RD}$, Muller WJ. Amplification of the neu/erbB-2 oncogene in a mouse model of mammary tumorigenesis. Proc Natl Acad Sci USA. 2000;97:3444-9.

9. Rosner A, Miyoshi K, Landesman-Bollag E, Xu X, Seldin DC, Moser AR, et al. Pathway pathology: histological differences between ErbB/Ras and Wnt pathway transgenic mammary tumors. Am J Pathol. 2002;161:1087-97.

10. Andrechek ER, Laing MA, Girgis-Gabardo AA, Siegel PM, Cardiff RD, Muller WJ. Gene expression profiling of neu-induced mammary tumors from transgenic mice reveals genetic and morphological similarities to ErbB2-expressing human breast cancers. Cancer Res 2003;63:4920-6.

11. Theodorou V, Kimm MA, Boer M, Wessels L, Theelen W, Jonkers J, et al. MMTV insertional mutagenesis identifies genes, gene families and pathways involved in mammary cancer. Nat Genet 2007;39:759-69.

12. Kim HH, van den Heuvel APJ, Schmidt JW, Ross SR. Novel common integration sites targeted by mouse mammary tumor virus insertion in mammary tumors have oncogenic activity. PLoS One 2011;6:e27425.

13. Callahan R, Mudunur U, Bargo S, Raafat A, McCurdy D, Boulanger $\mathrm{C}$, et al. Genes affected by mouse mammary tumor virus 
(MMTV) proviral insertions in mouse mammary tumors are deregulated or mutated in primary human mammary tumors. Oncotarget. 2012;3:1320-34.

14. Klijn C, Koudijs MJ, Kool J, ten Hoeve J, Boer M, de Moes J, et al. Analysis of tumor heterogeneity and cancer gene networks using deep sequencing of MMTV-induced mouse mammary tumors. PLoS One 2013;8:e62113.

15. Ikink GJ, Boer M, Bakker ERM, Hilkens J. IRS4 induces mammary tumorigenesis and confers resistance to HER2-targeted therapy through constitutive PI3K/AKT-pathway hyperactivation. Nat Commun. 2016;7:13567.

16. Nusse R. Insertional mutagenesis in mouse mammary tumorigenesis. In: Kung H-J, Vogt P, editors. Retroviral insertion and oncogene activation. Berlin: Springer; 1991. p. 43-65.

17. Hilkens J. Recent translational research: oncogene discovery by insertional mutagenesis gets a new boost. Breast Cancer Res 2006;8:102.

18. Callahan R, Smith GH. Common integration sites for MMTV in viral induced mouse mammary tumors. J Mammary Gland Biol Neoplasia 2008;13:309-21.

19. Koudijs MJ, Klijn C, Van Der Weyden L, Kool J, Ten Hoeve J, Sie D, et al. High-throughput semiquantitative analysis of insertional mutations in heterogeneous tumors. Genome Res 2011;21:2181-9.

20. Miyoshi J, Kagimoto M, Soeda E, Sakaki Y. The human c-Haras2 is a processed pseudogene inactivated by numerous base substitutions. Nucleic Acids Res 1984;12:1821-8.

21. Takahashi K, Mitsui K, Yamanaka S, Role of ERas in promoting tumour-like properties in mouse embryonic stem cells. Nature. 2003;423:541-5.

22. Polo JM, Anderssen E, Walsh RM, Schwarz BA, Nefzger CM, Lim SM, et al. A molecular roadmap of reprogramming somatic cells into iPS cells. Cell. 2012;151:1617-32.

23. Yu Y, Liang D, Tian Q, Chen X, Jiang B, Chou BK, et al. Stimulation of somatic cell reprogramming by ERas-Akt-FoxO1 signaling axis. Stem Cells 2014;32:349-63.

24. Zhao Z-A, Yu Y, Ma H-X, Wang X-X, Lu X, Zhai Y, et al. The roles of ERAS during cell lineage specification of mouse early embryonic development. Open Biol. 2015; 5: 150092. https://doi. org/10.1098/rsob.150092.

25. Akagi K, Suzuki T, Stephens RM, Jenkins NA, Copeland NG. RTCGD: retroviral tagged cancer gene database. Nucleic Acids Res 2004;32:D523-27.

26. Ikink GJ, Hilkens J, Insulin receptor substrate 4 (IRS4) is a constitutive active oncogenic driver collaborating with HER 2 and causing therapeutic resistance. Mol Cell Oncol. 2017;4:e1279722.

27. Holbro T, Beerli RR, Maurer F, Koziczak M, Barbas CF, Hynes NE. The ErbB2/ErbB3 heterodimer functions as an oncogenic unit: ErbB2 requires ErbB3 to drive breast tumor cell proliferation. Proc Natl Acad Sci Usa 2003;100:8933-8.

28. Lee-Hoeflich ST, Crocker L, Yao E, Pham T, Munroe X, Hoeflich $\mathrm{KP}$, et al. A central role for HER3 in HER2-amplified breast cancer: implications for targeted therapy. Cancer Res 2008;68:5878-87.

29. Ciriello G, Gatza ML, Beck AH, Wilkerson MD, Rhie SK, Pastore A, et al. Comprehensive molecular portraits of invasive lobular breast cancer. Cell. 2015;163:506-19.

30. Pereira B, Chin S-F, Rueda OM, Vollan H-KM, Provenzano E, Bardwell HA, et al. The somatic mutation profiles of 2,433 breast cancers refines their genomic and transcriptomic landscapes. Nat Commun 2016;7:11479.

31. Berns K, Horlings HM, Hennessy BT, Madiredjo M, Hijmans $\mathrm{EM}$, Beelen $\mathrm{K}$, et al. A functional genetic approach identifies the $\mathrm{PI} 3 \mathrm{~K}$ pathway as a major determinant of trastuzumab resistance in breast cancer. Cancer Cell. 2007;12:395-402.
32. Eichhorn PJA, Gili M, Scaltriti M, Serra V, Guzman M, Nijkamp $\mathrm{W}$, et al. Phosphatidylinositol 3-kinase hyperactivation results in lapatinib resistance that is reversed by the mTOR/phosphatidylinositol 3-kinase inhibitor NVP-BEZ235. Cancer Res 2008;68:9221-30.

33. Majewski IJ, Nuciforo P, Mittempergher L, Bosma AJ, Eidtmann $\mathrm{H}$, Holmes E, et al. PIK3CA mutations are associated with decreased benefit to neoadjuvant human epidermal growth factor receptor 2-targeted therapies in breast cancer. J Clin Oncol 2015;33:1334-9.

34. Wang PY, Seabold GK, Wenthold RJ. Synaptic adhesion-like molecules (SALMs) promote neurite outgrowth. Mol Cell Neurosci. 2008;39:83-94.

35. Mah W, Ko J, Nam J, Han K, Chung WS, Kim E. Selected SALM (synaptic adhesion-like molecule) family proteins regulate synapse formation. J Neurosci. 2010;30:5559-68.

36. Vyazunova I, Maklakova VI, Berman S, De I, Steffen MD, Hong W, et al. Sleeping Beauty Mouse Models Identify Candidate Genes Involved in Gliomagenesis. PLoS ONE. 2014;9:e113489..

37. Colicelli J. Human RAS superfamily proteins and related GTPases. Sci Stke 2004;2004:RE13.

38. Kameda T, Thomson JA. Human ERas gene has an upstream premature polyadenylation signal that results in a truncated, noncoding transcript. Stem Cells 2005;23:1535-40.

39. Kaizaki R, Yashiro M, Shinto O, Yasuda K, Matsuzaki T, Sawada $\mathrm{T}$, et al. Expression of ERas oncogene in gastric carcinoma. Anticancer Res 2009;29:2189-93.

40. Kubota E, Kataoka H, Aoyama M, Mizoshita T, Mori Y, Shimura $\mathrm{T}$, et al. Role of ES cell-expressed Ras (ERas) in tumorigenicity of gastric cancer. Am J Pathol. 2010;177:955-63.

41. Hynes NE, MacDonald G. ErbB receptors and signaling pathways in cancer. Curr Opin Cell Biol 2009;21:177-84.

42. Knutson KL, Almand B, Dang Y, Disis ML. neu Antigennegative variants can be generated after neu-specific antibody therapy in neu transgenic mice. Cancer Res 2004;64:1146-51.

43. Ellis MJ, Perou CM. The genomic landscape of breast cancer as a therapeutic roadmap. Cancer Discov 2013;3:27-34.

44. Nagata Y, Lan K-H, Zhou X, Tan M, Esteva FJ, Sahin AA, et al. PTEN activation contributes to tumor inhibition by trastuzumab, and loss of PTEN predicts trastuzumab resistance in patients. Cancer Cell. 2004;6:117-27.

45. Esteva FJ, Guo H, Zhang S, Santa-Maria C, Stone S, Lanchbury JS, et al. PTEN, PIK3CA, p-AKT, and p-p70S6K status: association with trastuzumab response and survival in patients with HER2-positive metastatic breast cancer. Am J Pathol. 2010;177:1647-56.

46. Wang L, Zhang Q, Zhang J, Sun S, Guo H, Jia Z, et al. PI3K pathway activation results in low efficacy of both trastuzumab and lapatinib. BMC Cancer. 2011;11:248.

47. Chandarlapaty S, Sakr RA, Giri D, Patil S, Heguy A, Morrow M, et al. Frequent mutational activation of the PI3K-AKT pathway in trastuzumab-resistant breast cancer. Clin Cancer Res 2012;18:6784-91.

48. Hanker AB, Pfefferle AD, Balko JM, Kuba MG, Young CD, Sánchez V, et al. Mutant PIK3CA accelerates HER2-driven transgenic mammary tumors and induces resistance to combinations of anti-HER2 therapies. Proc Natl Acad Sci USA. 2013;110:14372-7.

49. Aoyama M, Kataoka H, Kubota E, Tada T, Asai K. Resistance to chemotherapeutic agents and promotion of transforming activity mediated by embryonic stem cell-expressed Ras (ERas) signal in neuroblastoma cells. Int J Oncol 2010;37:1011-6.

50. Kubota E, Kataoka H, Tanaka M, Okamoto Y, Ebi M, Hirata Y, et al. ERas enhances resistance to CPT-11 in gastric cancer. Anticancer Res 2011;31:3353-60. 